\title{
Pacific
}

Journal of

Mathematics

\section{UNIQUENESS OF THE FOLIATION OF CONSTANT MEAN CURVATURE SPHERES IN ASYMPTOTICALLY FLAT 3-MANIFOLDS}

SHIGUANG MA 


\title{
UNIQUENESS OF THE FOLIATION OF CONSTANT MEAN CURVATURE SPHERES IN ASYMPTOTICALLY FLAT 3-MANIFOLDS
}

\author{
SHIGUANG MA
}

\begin{abstract}
This paper studies the constant mean curvature surface in asymptotically flat 3-manifolds with general asymptotics. Under some weak conditions, the foliation of stable spheres of constant mean curvature is shown to be unique outside some compact set in the asymptotically flat 3-manifold with positive mass.
\end{abstract}

\section{Introduction}

A three-manifold $M$ with a Riemannian metric $g$ and a two-tensor $K$ is called an initial data set $(M, g, K)$ if $g$ and $K$ satisfy the constraint equations

$$
R_{g}-|K|_{g}^{2}+\left(\operatorname{tr}_{g}(K)\right)^{2}=16 \pi \rho \quad \text { and } \quad \operatorname{div}_{g}(K)-d\left(\operatorname{tr}_{g}(K)\right)=8 \pi J,
$$

where $R_{g}$ is the scalar curvature of the metric $g, \operatorname{tr}_{g}(K)$ denotes $g^{i j} K_{i j}, \rho$ is the observed energy density, and $J$ is the observed momentum density.

Definition 1.1. Let $q \in\left(\frac{1}{2}, 1\right]$. An initial data set $(M, g, K)$ is called an asymptotically flat (AF) manifold if there is a compact subset $\tilde{K} \subset M$ and coordinates $\left\{x^{i}\right\}$ with the following properties: $M \backslash \tilde{K}$ is diffeomorphic to $\mathbb{R}^{3} \backslash B_{1}(0) ; \rho$ and $J$ from (1-1) satisfy $\rho(x)=O\left(|x|^{-2-2 q}\right)$ and $J(x)=O\left(|x|^{-2-2 q}\right)$; and

$$
g_{i j}(x)=\delta_{i j}+h_{i j}(x) \quad \text { with } \quad h_{i j}(x)=O_{5}\left(|x|^{-q}\right) K_{i j}(x)=O_{1}\left(|x|^{-1-q}\right),
$$

where $f=O_{k}\left(|x|^{-q}\right)$ means $\partial^{l} f=O\left(|x|^{-l-q}\right)$ for $l=0, \ldots, k$. We call $M \backslash \tilde{K}$ an end of the AF manifold $(M, g, K)$; we will only consider AF manifolds with one end. The mass of this end is defined as

$$
m=\lim _{r \rightarrow \infty} \frac{1}{16 \pi} \int_{|x|=r}\left(h_{i j, j}-h_{j j, i}\right) v_{g}^{i} d \mu_{g},
$$

where $v_{g}$ and $d \mu_{g}$ are the unit normal vector and volume form with respect to the metric $g$. From [Bartnik 1986], we know the mass is well defined when $q>\frac{1}{2}$.

MSC2000: primary 53C20; secondary 58E20, 83C99.

Keywords: constant mean curvature, foliation, asymptotically flat manifold. 
Given a function $f$, let $f^{\text {odd }}(x)=f(x)-f(-x)$ and $f^{\text {even }}(x)=f(x)+f(-x)$.

Definition 1.2. An AF manifold $(M, g, K)$ is said to satisfy the Regge-Teitelboim condition, and is called an AF-RT manifold, if $\rho$ and $J$ satisfy

$$
\rho^{\text {odd }}(x)=O\left(|x|^{-3-2 q}\right), \quad J^{\text {odd }}(x)=O\left(|x|^{-3-2 q}\right)
$$

and $g, K$ satisfy the asymptotically even/odd conditions

$$
h_{i j}^{\text {odd }}(x)=O_{2}\left(|x|^{-1-q}\right), \quad K_{i j}^{\text {even }}(x)=O_{1}\left(|x|^{-2-q}\right) .
$$

For AF-RT manifolds, the center of mass $C$ is defined by

$$
C^{\alpha}=\frac{1}{16 \pi m} \lim _{r \rightarrow \infty}\left(\int_{|x|=r} x^{\alpha}\left(h_{i j, i}-h_{i i, j}\right) v_{g}^{j} d \mu_{g}-\int_{|x|=r}\left(h_{i \alpha} v_{g}^{i}-h_{i i} v_{g}^{\alpha}\right) d \mu_{g}\right) .
$$

From [Huang 2009], we know the center of mass is well defined.

Let $\Sigma$ be a surface of constant mean curvature (CMC). We say that $\Sigma$ is stable if the second variation operator has only nonnegative eigenvalues when restricted to the functions with 0 mean value, that is,

$$
\int_{\Sigma}\left(|A|^{2}+\operatorname{Ric}\left(v_{g}, v_{g}\right)\right) f^{2} d \mu \leq \int_{\Sigma}|\nabla f|^{2} d \mu
$$

for $f$ a function with $\int_{\Sigma} f d \mu=0$, where $A$ is the second fundamental form and $\operatorname{Ric}\left(v_{g}, v_{g}\right)$ is the Ricci curvature in the normal direction with respect to the metric $g$.

We discuss the existence and uniqueness of $\mathrm{CMC}$ spheres that separate the compact part from infinity in AF-RT manifolds. The following two theorems are due to Lan-Hsuan Huang [2010]:

Theorem 1.3 (existence). If $(M, g, K)$ is AF-RT with $q \in\left(\frac{1}{2}, 1\right]$ and $m>0$, there exists a foliation by spheres $\left\{\Sigma_{R}\right\}$ with constant mean curvature $H\left(\Sigma_{R}\right)=$ $2 / R+O\left(R^{-1-q}\right)$ in the exterior region of $M$. Each leaf $\Sigma_{R}$ is a $\left(c_{0} R^{1-q}\right)$-graph over $S_{R}(C)$ and is strictly stable.

Set $r(x)=\left(\sum\left(x^{i}\right)^{2}\right)^{1 / 2}$. For a CMC sphere $\Sigma$ separating infinity from $\tilde{K}$, define

$$
r_{0}(\Sigma)=\inf \{r(x) \mid x \in \Sigma\}, \quad r_{1}(\Sigma)=\sup \{r(x) \mid x \in \Sigma\} .
$$

Theorem 1.4 (uniqueness). Assume that $(M, g, K)$ is $A F-R T$ with $q \in\left(\frac{1}{2}, 1\right]$ and $m>0$. There exist $\sigma_{1}$ and $C_{1}$ such that, if $\Sigma$ is a topological sphere of constant mean curvature $H=H\left(\Sigma_{R}\right)$ for some $R \geq \sigma_{1}$, and moreover $\Sigma$ is stable and satisfies $r_{1}(\Sigma) \leq C_{1} r_{0}^{1 / a}$ for some $a \in\left(\frac{5-q}{2(2+q)}, 1\right]$, then $\Sigma=\Sigma_{R}$. 
Uniqueness is the harder problem. In Theorem 1.4, Huang needs the assumption $r_{1} \leq C_{1} r_{0}^{1 / a}$ for the radius of the surface. To get a sharper uniqueness result as in [Qing and Tian 2007], we consider metrics of the following form:

Definition 1.5. An AF-RT manifold $(M, g, K)$ with mass $m$ is called an $(m, k, \varepsilon)$ $A F-R T$ manifold, where $k>2$ and $\varepsilon>0$, if the metric $g$ can be expressed as

$$
g_{i j}=\delta_{i j}+h_{i j}=\delta_{i j}+h_{i j}^{1}(\theta) / r+Q,
$$

with $h_{i j}^{1}(\theta) \in C^{5}\left(S^{2}\right), Q=O_{5}\left(|x|^{-2}\right)$, and

$$
\left\|h_{i j}^{1}(\theta)-\delta_{i j}(\theta)\right\|_{W^{k, 2}\left(S^{2}\right)} \leq \varepsilon .
$$

Here $\theta=\left(\theta_{1}, \theta_{2}\right)$ is the coordinate on $S^{2} \subset \mathbb{R}^{3}$.

Remark 1.6. From (1-3), we know that the mass $m$ has positive two-side bounds. We can certainly consider the case $\left\|h_{i j}^{1}(\theta)-C \delta_{i j}(\theta)\right\|_{W^{k, 2}\left(S^{2}\right)} \leq \varepsilon$ for any positive $C$, but here we only assume $C=1$ without lost of generality.

Our main uniqueness result is this:

Theorem 1.7. For any $k>2$ there exists $\varepsilon>0$, depending only on $k$, with the following property. For any $(m, k, \varepsilon)-A F-R T$ manifold $(M, g, K)$, there exists a compact $\tilde{K}$ and a constant $C>0$ such that, for any constant $H>0$ sufficiently small, there is a unique stable CMC sphere $\Sigma$ separating $\tilde{K}$ from infinity and such that $H(\Sigma)=H$ and $\log r_{1}(\Sigma) \leq C r_{0}(\Sigma)^{1 / 4}$.

Remark 1.8. This is an improvement on Huang's result, as can be seen by comparing $r_{1} \leq C_{1} r_{0}^{1 / a}$ with $\log r_{1}(\Sigma) \leq C r_{0}(\Sigma)^{1 / 4}$ (since $\log r_{1}$ grows much more slowly than any positive power of $r_{1}$ ).

Remark 1.9. In the proof of Theorem 1.7 , the RT condition is needed only for the existence theorem in [Huang 2010].

Remark 1.10. Here I can only deal with the case when $q=1$. When $q \in\left(\frac{1}{2}, 1\right)$ it seems that $\left\|h_{i j}(\theta)-\delta_{i j}(\theta)\right\|_{W^{k, 2}\left(S^{2}\right)} \leq \varepsilon$ is not a proper condition.

The paper is organized much like [Qing and Tian 2007]: In Section 2 we give an a priori estimate on stable CMC spheres based on Simon's identity. In Section 3, we introduce blow-down analysis in three different scales. In Section 4 we recall the asymptotic analysis from [Qing and Tian 1997] and prove a technical lemma. In Section 5 we introduce asymptotically harmonic coordinates. In Section 6 we introduce the notion of the center of mass and prove Theorem 1.7. 


\section{Acknowledgments}

I would like to thank my advisor, Prof. Gang Tian, for suggesting this problem to me and for many ideas, including the use of harmonic coordinates. I also thank Prof. Jie Qing and Prof. Yuguang Shi for useful discussions. I was partially supported by the China Scholarship Council.

\section{Curvature estimates}

In this section and the next we assume that $(M, g, K)$ is AF-RT with $q \in\left(\frac{1}{2}, 1\right]$. Let $\Sigma$ be a CMC sphere in the asymptotically flat end $(M \backslash \tilde{K}, g)$, and assume $\Sigma$ separates the compact part from infinity. First we have the following estimate, similar to [Huisken and Yau 1996, Lemma 5.2].

Lemma 2.1. Let $X=x^{i}\left(\partial / \partial x^{i}\right)$ be the Euclidean coordinate vector field and

$$
r=\left(\sum\left(x^{i}\right)^{2}\right)^{1 / 2} .
$$

With respect to the metric $g$, let $v$ be the outward normal vector field, $d \mu$ be the volume form of $\Sigma$. Then we have the estimate

$$
\int_{\Sigma}\langle X, v\rangle^{2} r^{-4} d \mu \leq H^{2}|\Sigma| .
$$

Moreover for each $a \geq a_{0}>2$ and $r_{0}$ sufficiently large,

$$
\int_{\Sigma} r^{-a} d \mu \leq C\left(a_{0}\right) r_{0}^{2-a} H^{2}|\Sigma| \text {. }
$$

Proof. Because the mean curvature $H$ is constant, for some smooth vector field $Y$ on $\Sigma$, we have the divergence formula

$$
\int_{\Sigma} \operatorname{div}_{\Sigma} Y d \mu=H \int_{\Sigma}\langle Y, v\rangle d \mu
$$

Choose $Y=X r^{-a}, a \geq 2$ and let $e_{\alpha}$ be the orthonormal basis on $\Sigma, \alpha=1,2$. Supposing $e_{\alpha}=a_{\alpha}^{i}\left(\partial / \partial x^{i}\right)$, it is obvious that $a_{\alpha}^{i}$ is bounded because the manifold is asymptotically flat. Then

$$
\begin{aligned}
\operatorname{div}_{\Sigma} Y & =\operatorname{div}_{\Sigma}\left(X r^{-a}\right)=\left\langle\nabla_{e_{\alpha}}\left(X r^{-a}\right), e_{\alpha}\right\rangle \\
& =r^{-a} \operatorname{div}_{\Sigma} X-a r^{-a-2} a_{\alpha}^{i} a_{\alpha}^{j} x^{i} x^{j}+O\left(r^{-a-q}\right) \\
& =r^{-a} \operatorname{div}_{\Sigma} X-\alpha r^{-a-2}\left|X^{\tau}\right|^{2}+O\left(r^{-a-q}\right),
\end{aligned}
$$

where $X^{\tau}$ is the tangent projection of $X$. Also,

$$
\left|\operatorname{div}_{\Sigma} X-2\right|=O\left(r^{-q}\right) .
$$


Note that $\left|X^{\tau}\right|^{2}=r^{2}-\langle X, v\rangle^{2}+O\left(r^{2-q}\right)$. Combining all of these,

$$
\begin{aligned}
\left|(2-a) \int_{\Sigma} r^{-a} d \mu+a \int_{\Sigma}\langle X, v\rangle^{2} r^{-a-2} d \mu-H \int_{\Sigma}\langle X, v\rangle r^{-a} d \mu\right| \\
\leq C \int_{\Sigma} r^{-a-q} d \mu .
\end{aligned}
$$

Choosing $a=2$, from Hölder's inequality, we have

$$
\int_{\Sigma}\langle X, v\rangle^{2} r^{-4} d \mu \leq \frac{1}{4} H^{2}|\Sigma|+C \int_{\Sigma} r^{-2-q} d \mu .
$$

Then choosing $a=2+q$ gives

$$
\int_{\Sigma} r^{-2-q} d \mu \leq 4 r_{0}^{-q}\left(\int_{\Sigma}\langle X, v\rangle^{2} r^{-4} d \mu+H^{2}|\Sigma|+C \int_{\Sigma} r^{-2-q} d \mu\right) .
$$

This combined with (2-2) implies

$$
\int_{\Sigma}\langle X, v\rangle^{2} r^{-4} d \mu \leq H^{2}|\Sigma|
$$

Again from (2-1), we have for $a \geq a_{0}>2$,

$$
\int_{\Sigma} r^{-a} \leq C\left(a_{0}-2\right)^{-1} r_{0}^{2-a} H^{2}|\Sigma|
$$

Now we can derive the integral estimate for $|\AA|$ from the stability of the surface as in [Huisken and Yau 1996, Proposition 5.3]:

Lemma 2.2. Suppose $\Sigma$ is a stable CMC sphere in an asymptotically flat manifold. For $r_{0}$ sufficiently large,

$$
\begin{aligned}
\int_{\Sigma}|\AA|^{2} d \mu & \leq C r_{0}^{-q} \\
H^{2}|\Sigma| & \leq C \\
\int_{\Sigma} H^{2} d \mu & =16 \pi+O\left(r_{0}^{-q}\right) .
\end{aligned}
$$

Proof. Since $\Sigma$ is stable,

$$
\int_{\Sigma}|\nabla f|^{2} d \mu \geq \int_{\Sigma}\left(|A|^{2}+\operatorname{Ric}(v, v)\right) f^{2} d \mu
$$

for any function $f$ with $\int_{\Sigma} f d \mu=0$, where $A$ is the second fundamental form of $\Sigma$ and Ric is the Ricci curvature of $M$.

Choose $\psi$ to be a conformal map of degree 1 from $\Sigma$ to the standard $S^{2}$ in $\mathbb{R}^{3}$. Each component $\psi_{i}$ of $\psi$ can be chosen such that $\int \psi_{i} d \mu=0$ [Li and Yau 1982]. 
For each $\psi_{i}$,

$$
\int_{\Sigma}\left|\nabla \psi_{i}\right|^{2} d \mu=\frac{8 \pi}{3}
$$

Since $\sum \psi_{i}^{2} \equiv 1$ we conclude that

$$
\int_{\Sigma}|A|^{2}+\operatorname{Ric}(v, v) d \mu \leq 8 \pi
$$

From the Gauss equation

$$
\frac{1}{2}|A|^{2}+\operatorname{Ric}(v, v)-\frac{1}{2} R+K=\frac{1}{2} H^{2},
$$

we have

$$
|A|^{2}+\operatorname{Ric}(v, v)=\frac{1}{2}|\AA|^{2}+\frac{3}{4} H^{2}+\frac{1}{2} R-K,
$$

where $K$ is the Gauss curvature of $\Sigma$ and $\AA$ is defined as $\AA_{i j}=A_{i j}-(H / 2) g_{i j}$.

Then

$$
\int_{\Sigma} \frac{1}{2}|\AA|^{2}+\frac{3}{4} H^{2}|\Sigma| \leq 12 \pi+r_{0}^{-q} H^{2}|\Sigma|
$$

because $R=O\left(r^{-2-2 q}\right)$, from the constraint equation (1-1). So $H^{2}|\Sigma| \leq 16 \pi$.

Using the Gauss equation in a different way, we have

$$
\begin{aligned}
\int_{\Sigma}|\AA|^{2} d \mu & =\int_{\Sigma}|A|^{2}-\frac{H^{2}}{2} d \mu \\
& =\frac{1}{2} \int_{\Sigma}|A|^{2}+\operatorname{Ric}(v, v) d \mu+\frac{1}{2} \int_{\Sigma} R-3 \operatorname{Ric}(v, v)-2 K d \mu \\
& \leq \int_{\Sigma} r^{-2-q} d \mu \\
& =O\left(r_{0}^{-q}\right) .
\end{aligned}
$$

Then again from the Gauss equation (2-3),

$$
\int_{\Sigma} H^{2} d \mu=4 \int_{\Sigma} K d \mu+O\left(r_{0}^{-q}\right)=16 \pi+O\left(r_{0}^{-q}\right) .
$$

Lemma 2.3. Suppose $M$ is a constant mean curvature surface in an asymptotically flat end $\left(\mathbb{R}^{3} \backslash B_{1}(0), g\right)$. Then

$$
\int_{\Sigma} H_{e}^{2} d \mu_{e}=16 \pi+O\left(r_{0}^{-q}\right)
$$

Proof. We follow the calculation of Huisken and Ilmanen [2001] to obtain

$$
g_{i j}=\delta_{i j}+h_{i j}
$$


Suppose that

$$
\left.g_{i j}\right|_{\Sigma}=f_{i j},\left.\quad \delta_{i j}\right|_{\Sigma}=\varepsilon_{i j},
$$

where $f^{i j}$ and $\epsilon^{i j}$ are the corresponding inverse matrices. Let $v, \omega, A, H, d \mu$ represent the normal vector, the dual form of $v$, the second fundamental form, the mean curvature and the volume form of $\Sigma$ in the metric $g$ and $v_{e}, \omega_{e}, A_{e}, H_{e}, \mu_{e}$ represent the corresponding ones in Euclidean metric. Easy calculation gives

$$
\begin{aligned}
f^{i j}-\varepsilon^{i j} & =-f^{i k} h_{k l} f^{l j} \pm C|h|^{2}, \\
g^{i j}-\delta^{i j} & =-g^{i k} h_{k l} g^{l j} \pm C|h|^{2}, \\
\omega & =\frac{\omega_{e}}{\left|\omega_{e}\right|}, \quad v^{i}=g^{i j} \omega_{j}, \\
\left(\omega_{e}\right)_{i} & =\omega_{i} \pm C|P|, \quad v_{e}^{i}=v^{i}+C|h|, \quad 1-\left|\omega_{e}\right|=\frac{1}{2} h_{i j} v^{i} v^{j}, \\
\Gamma_{i j}^{k} & =\frac{1}{2} g^{k l}\left(\bar{\nabla}_{i} h_{j l}+\bar{\nabla}_{j} h_{i l}-\bar{\nabla}_{l} h_{i j}\right) \pm C|h| \pm C|\bar{\nabla} h|,
\end{aligned}
$$

where $\Gamma_{i j}^{k}$ is the Christoffel symbol for $\bar{\nabla}-\bar{\nabla}_{e}$ and we denote the gradient for the metrics $g$ and $\delta$ by $\bar{\nabla}$ and $\bar{\nabla}_{e}$.

We have the formula

$$
\left|\omega_{e}\right|_{g} A_{i j}=\left(A_{e}\right)_{i j}-\left(\omega_{e}\right)_{k} \Gamma_{i j}^{k}
$$

This implies

$$
\begin{aligned}
H-H_{e} & =f^{i j} A_{i j}-\varepsilon^{i j}\left(A_{e}\right)_{i j} \\
& =\left(f^{i j}-\varepsilon^{i j}\right) A_{i j}+\varepsilon^{i j} A_{i j}\left(1-\left|\omega_{e}\right|_{g}\right)+\varepsilon^{i j}\left(\left|\omega_{e}\right|_{g} A_{i j}-\left(A_{e}\right)_{i j}\right) .
\end{aligned}
$$

From (2-4), (2-5), (2-7),

$$
\varepsilon^{i j} A_{i j}\left(1-\left|\omega_{e}\right|_{g}\right)=\frac{1}{2} H v^{i} v^{j} h_{i j} \pm C|h|^{2}|A| .
$$

Using (2-4)-(2-9), we obtain

$$
\begin{aligned}
\varepsilon^{i j}\left(\left|\omega_{e}\right| A_{i j}-\left(A_{e}\right)_{i j}\right) & =-\varepsilon^{i j}\left(\omega_{e}\right)_{k} \Gamma_{i j}^{k} \\
& =-\frac{1}{2} f^{i j} \omega_{k} g^{k l}\left(\bar{\nabla}_{i} h_{j l}+\bar{\nabla}_{j} h_{i l}-\bar{\nabla}_{l} h_{i j}\right) \pm C|h||\bar{\nabla} h| \\
& =-f^{i j} v^{l} \bar{\nabla}_{i} h_{j l}+\frac{1}{2} f^{i j} v^{l} \bar{\nabla}_{l} h_{i j} \pm C|h||\bar{\nabla} h| .
\end{aligned}
$$

At last,

$$
\begin{aligned}
H-H_{e}=-f^{i k} h_{k l} f^{l j} A_{i j}+\frac{1}{2} H & v^{i} v^{j} h_{i j}-f^{i j} v^{l} \bar{\nabla}_{i} h_{j l} \\
& +\frac{1}{2} f^{i j} v^{l} \bar{\nabla}_{l} h_{i j} \pm C|h||\bar{\nabla} h| \pm C|h|^{2}|A|,
\end{aligned}
$$


and

$$
\begin{aligned}
\int_{\Sigma} H_{e}^{2} d \mu_{e} & =\left(1+O\left(r_{0}^{-q}\right)\right) \int_{\Sigma} H_{e}^{2} d \mu \\
\leq & \left(1+O\left(r_{0}^{-q}\right)\right)\left(\int_{\Sigma} H^{2} d \mu+\int_{\Sigma}\left(H_{e}-H\right)^{2}+2\left|H\left(H_{e}-H\right)\right| d \mu\right) \\
\leq\left(1+O\left(r_{0}^{-q}\right)\right)\left(16 \pi+O\left(r_{0}^{-q}\right)+\int_{\Sigma}\left(H_{e}-H\right)^{2}\right. & \left.\quad+\left(\int_{\Sigma} H^{2} d \mu\right)^{1 / 2}\left(\int_{\Sigma}\left(H_{e}-H\right)^{2} d \mu\right)^{1 / 2}\right) \\
\int\left(H_{e}-H\right)^{2} d \mu & \leq \int O\left(|x|^{-2 q}\right)|A|^{2}+H^{2} O\left(|x|^{-2 q}\right)+O\left(|x|^{-2-2 q}\right) d \mu \\
& \leq \int O\left(|x|^{-2 q}\right) H^{2}+O\left(|x|^{-2 q}\right)|\AA|^{2}+O\left(|x|^{-2-2 q}\right) d \mu \\
& =O\left(r_{0}^{-2 q}\right),
\end{aligned}
$$

so we have

$$
\int_{\Sigma} H_{e}^{2} d \mu_{e} \leq 16 \pi+O\left(r_{0}^{-q}\right)
$$

On the other hand, by Euler's formula,

$$
K_{e}=\frac{1}{4} H_{e}^{2}-\frac{1}{2}\left|\AA_{e}\right|^{2} .
$$

So we have

which implies

$$
\int H_{e}^{2} d \mu_{e} \geq 16 \pi
$$

$$
\int_{\Sigma} H_{e}^{2} d \mu_{e}=16 \pi+O\left(r_{0}^{-q}\right)
$$

Based on [Michael and Simon 1973] we have the following Sobolev inequality.

Lemma 2.4. Suppose $\Sigma$ is a constant mean curvature surface in an asymptotically flat end $\left(\mathbb{R}^{3} \backslash B_{1}(0), g\right)$ with $r_{0}(\Sigma)$ sufficiently large and that $\int_{\Sigma} H^{2} \leq C$. Then

$$
\left(\int_{\Sigma} f^{2} d \mu\right)^{1 / 2} \leq C\left(\int_{\Sigma}|\nabla f| d \mu+\int_{\Sigma} H|f| d \mu\right) .
$$

Proof. Note that this is valid for a surface in Euclidean space. So by the uniform equivalence of the metrics $g$ and $\delta$, we have

$$
\left(\int|f|^{2} d \mu\right)^{1 / 2} \leq C\left(\int|f|^{2} d \mu_{e}\right)^{1 / 2} \leq C\left(\int|\nabla f|+H|f|+\left|H-H_{e}\right||f| d \mu\right) .
$$


To bound the last term on the right, we use

$$
\begin{aligned}
\int\left|H-H_{e}\right||f| d \mu & \leq \int O\left(|x|^{-q}\right)|A||f|+O\left(|x|^{-q}\right) H|f|+O\left(|x|^{-1-q}\right)|f| d \mu \\
& \leq O\left(r_{0}^{-q}\right) \int H|f|+\left(\int|\AA|^{2} d \mu\right)^{1 / 2} O\left(r_{0}^{-q}\right)\|f\|_{L^{2}} \\
& +O\left(r_{0}^{-q}\right)\|f\|_{L^{2}} .
\end{aligned}
$$

So we can choose $r_{0}$ sufficiently large to get the desired result.

Lemma 2.5. Suppose $\Sigma$ is a constant mean curvature surface in an asymptotically flat end $\left(\mathbb{R}^{3} \backslash B_{1}(0), g\right)$ with $r_{0}(\Sigma)$ sufficiently large. Then

$$
C_{1} H^{-1} \leq \operatorname{diam}(\Sigma) \leq C_{2} H^{-1} .
$$

where diam $(\Sigma)$ denotes the diameter of $\Sigma$ in the Euclidean space $\mathbb{R}^{3}$. In particular, if the surface $\Sigma$ separates infinity from the compact part, then

$$
C_{1} H^{-1} \leq r_{1}(\Sigma) \leq C_{2} H^{-1} \text {. }
$$

Proof. We already know that

$$
\int_{\Sigma} H_{e}^{2} d \mu_{e}=16 \pi+O\left(r_{0}^{-q}\right)
$$

Then from [Simon 1993, Lemma 1.1],

$$
\sqrt{\frac{2|\Sigma|_{e}}{F(\Sigma)}} \leq \operatorname{diam}(\Sigma) \leq C \sqrt{|\Sigma|_{e} F(\Sigma)},
$$

where $F(\Sigma)=\frac{1}{2} \int_{\Sigma} H_{e}^{2}$ is the Willmore functional and $|\Sigma|_{e}$ is the volume of $\Sigma$ with respect to the Euclidean metric. Since the Euclidean metric is uniformly equivalent to $g$, we get the result.

To get the pointwise estimate for $\AA$, we use Simon's identity (2-12) below and Moser's iteration argument.

Lemma 2.6 [Schoen et al. 1975]. Suppose $N$ is a hypersurface in a Riemannian manifold $(M, g)$. Then the second fundamental form satisfies the identity

$$
\begin{array}{r}
\Delta A_{i j}=\nabla_{i} \nabla_{j} H+H A_{i k} A_{j k}-|A|^{2} A_{i j}+H R_{3 i 3 j}-A_{i j} R_{3 k 3 k}+A_{j k} R_{k l i l} \\
+A_{i k} R_{k l j l}-2 A_{l k} R_{i l j k}+\bar{\nabla}_{j} R_{3 k i k}+\bar{\nabla}_{k} R_{3 i j k},
\end{array}
$$

where $R_{i j k l}$ and $\bar{\nabla}$ are the curvature and gradient operator of $(M, g)$. 
From this we easily deduce for CMC surfaces the inequality

$-|\AA| \Delta|\AA| \leq|\AA|^{4}+C H|\AA|^{3}+C H^{2}|\AA|^{2}+C|\AA|^{2}|x|^{-2-q}$

$$
+C H|\AA||x|^{-2-q}+C|\AA||x|^{-3-q} .
$$

We also need an inequality for $\nabla \AA$ because we also want to estimate the higher derivative:

$$
\begin{aligned}
& -|\nabla \AA| \Delta|\nabla \AA| \leq C|\nabla \AA|^{2}\left(|\AA|^{2}+H|\AA|+H^{2}+O\left(|x|^{-2-q}\right)\right) \\
& +|\nabla \AA|\left(\left(|\AA|^{2}+H|\AA|+H^{2}\right) O\left(|x|^{-2-q}\right)+(|\AA|+H) O\left(|x|^{-3-q}\right)+O\left(|x|^{-4-q}\right)\right) .
\end{aligned}
$$

Then we can get the pointwise estimates for $\AA$ and $\nabla \AA$.

Theorem 2.7 [Qing and Tian 2007]. Suppose that $\left(\mathbb{R}^{3} \backslash B_{1}(0), g\right)$ is an asymptotically flat end. Then there exist positive numbers $\sigma_{0}, \delta_{0}$ such that for any constant mean curvature surface in the end which separates infinity from the compact part, we have

$$
|\AA|^{2}(x) \leq C|x|^{-2} \int_{B_{\delta_{0}|x|}(x)}|\AA|^{2} d \mu+C|x|^{-2-2 q} \leq C|x|^{-2} r_{0}^{-q}
$$

and

$$
|\nabla \AA|^{2}(x) \leq C|x|^{-2} \int_{B_{\delta_{0}|x|}(x)}|\nabla \AA|^{2} d \mu+C|x|^{-4-2 q} \leq C|x|^{-4} r_{0}^{-q / 2},
$$

provided that $r_{0} \geq \sigma_{0}$.

Proof. In the Sobolev inequality (2-11), take $f=u^{2}$. Then

$$
\begin{aligned}
\left(\int_{\Sigma} u^{4} d \mu\right)^{1 / 2} & \leq C\left(2 \int_{\Sigma}|u||\nabla u| d \mu+\int_{\Sigma} H u^{2} d \mu\right) \\
& \leq C\left(\int_{\Sigma} u^{2}\right)^{1 / 2}\left(\int_{\Sigma}|\nabla u|^{2} d \mu\right)^{1 / 2}+C\left(\int_{\operatorname{supp}(u)} H^{2} d \mu\right)^{1 / 2}\left(\int_{\Sigma} u^{4} d \mu\right)^{1 / 2} .
\end{aligned}
$$

To proceed, we need some auxiliary results.

Lemma 2.8. For any $\varepsilon>0$, we can find a uniform $\delta_{0}$ sufficiently small such that

$$
\int_{B_{\delta_{0}|x|}(x)} H^{2} d \mu \leq \varepsilon \quad \text { for any } x \in \Sigma .
$$

Proof. The metric $g$ is equivalent to Euclidean metric $\delta$. Thus we need only to prove that there exists $C$ such that

$$
\left|B_{\delta_{0}|x|}(x)\right|_{e} \leq C \delta_{0}^{2}|x|^{2}
$$


because then

$$
H^{2}\left|B_{\delta_{0}|x|}(x)\right|_{e} \leq C \delta_{0}^{2}|x|^{2} H^{2} \leq C \delta_{0}^{2} .
$$

From the proof of Lemma 1.1 in [Simon 1993] we know that, for any $x \in \Sigma$, if $B_{\sigma}(x)$ denotes the Euclidean ball of radius $\sigma$ with center $x$ in $\mathbb{R}^{3}$ and $\Sigma_{\sigma}=$ $\Sigma \cap B_{\sigma}(x)$, then there exists $C$ such that for $0<\sigma \leq \rho<\infty$,

$$
\sigma^{-2}\left|\Sigma_{\sigma}\right|_{e} \leq C\left(\rho^{-2}\left|\Sigma_{\rho}\right|+F\left(\Sigma_{\rho}\right)\right),
$$

where $F\left(\Sigma_{\rho}\right)$ is the Willmore functional. The constant $C$ does not depend on $\Sigma, \sigma, \rho$.

Letting $\rho \rightarrow \infty, \rho^{-2}\left|\Sigma_{\rho}\right| \rightarrow 0$ gives

$$
\sigma^{-2}\left|\Sigma_{\sigma}\right|_{e} \leq C F(\Sigma) \leq C .
$$

This proves the lemma.

So if $\operatorname{supp}(u) \subset B_{\delta_{0}|x|}(x)$, we have the scaling invariant Sobolev inequality

$$
\left(\int_{\Sigma} u^{4} d \mu\right)^{1 / 2} \leq C\left(\int_{\Sigma} u^{2}\right)^{1 / 2}\left(\int_{\Sigma}|\nabla u|^{2} d \mu\right)^{1 / 2}
$$

Lemma 2.9 [Qing and Tian 2007, Lemma 2.6]. Suppose a nonnegative function $v \in L^{2}$ solves

$$
-\Delta v \leq f v+h
$$

on $B_{2 R}\left(x_{0}\right)$, where

$$
\int_{B_{2 R}\left(x_{0}\right)} f^{2} d \mu \leq C R^{-2}
$$

and $h \in L^{2}\left(B_{2 R}\left(x_{0}\right)\right)$. Also, suppose that

$$
\left(\int_{\Sigma} u^{4} d \mu\right)^{1 / 2} \leq C\left(\int_{\Sigma} u^{2}\right)^{1 / 2}\left(\int_{\Sigma}|\nabla u|^{2} d \mu\right)^{1 / 2}
$$

holds for all $u$ with support inside $B_{2 R}\left(x_{0}\right)$. Then

$$
\sup _{B_{R}\left(x_{0}\right)} v \leq C R^{-1}\|v\|_{L^{2}\left(B_{2 R}\left(x_{0}\right)\right)}+C R\|h\|_{L^{2}\left(B_{2 R\left(x_{0}\right)}\right)} .
$$

Then we find that

$$
\begin{aligned}
-\Delta|\AA| & \leq\left(|\AA|^{2}+H^{2}+H|\AA|+C|x|^{-2-q}\right)|\AA|+C H|x|^{-2-q}+C|x|^{-3-q} \\
& =f_{1}|\AA|+h_{1}
\end{aligned}
$$


and

$$
\begin{aligned}
-\Delta|\nabla \AA| \leq & C|\nabla \AA|\left(|\AA|^{2}+H|\AA|+H^{2}+O\left(|x|^{-3}\right)\right) \\
& +\left(\left(|\AA|^{2}+H|\AA|+H^{2}\right) O\left(|x|^{-3}\right)+(|\AA|+H) O\left(|x|^{-4}\right)+O\left(|x|^{-5}\right)\right) \\
= & f_{2}|\nabla \AA|+h_{2} .
\end{aligned}
$$

As in Theorem 2.5 of [Qing and Tian 2007] we have $\left\|f_{i}\right\|_{L^{2}\left(B_{2 \delta_{0}|x|}(x)\right)}^{2} \leq C|x|^{-2}$ for $i=1,2$. Further, it is easy to show that

$$
\left\|h_{1}\right\|_{L^{2}\left(B_{2 \delta_{0}|x|}(x)\right)}^{2}=O\left(|x|^{-4-2 q}\right) \quad \text { and } \quad\left\|h_{2}\right\|_{L^{2}\left(B_{2 \delta_{0}|x|}(x)\right)}^{2}=O\left(|x|^{-6-2 q}\right) .
$$

At last we know that

$$
\int_{B_{\delta_{0}|x|}(x)}|\AA|^{2} d \mu \leq C|x|^{-2} r_{0}^{-q},
$$

and

$$
\int_{B_{\delta_{0}|x|}(x)}|\nabla \AA|^{2} d \mu \leq|x|^{-2}\left(\int_{B_{\delta_{0}|x|}(x)}|\AA|^{2} d \mu\right)^{1 / 2} \leq|x|^{-2} r_{0}^{-q / 2} .
$$

The first inequality follows from Lemma 2.2 and the second one from Simon's identity (2-12). This concludes the proof of Theorem 2.7.

\section{Blow down analysis}

Now like [Qing and Tian 2007], we blow down the surface in three different scales. First we consider

$$
\tilde{N}=\frac{1}{2} H N=\left\{\frac{1}{2} H x \mid x \in N\right\} .
$$

Suppose there is a sequence of constant mean curvature surfaces $\left\{N_{i}\right\}$ such that

We know that

$$
\lim _{i \rightarrow \infty} r_{0}\left(N_{i}\right)=\infty
$$

$$
\lim _{i \rightarrow \infty} \int_{N_{i}} H_{e}^{2} d \mu_{e}=16 \pi .
$$

Hence, by the curvature estimates established in the previous section combined with the proof of [Simon 1993, Theorem 3.1], we have:

Lemma 3.1. Suppose that $\left\{N_{i}\right\}$ is a sequence of constant mean curvature surfaces in a given asymptotically flat end $\left(\mathbb{R}^{3} \backslash B_{1}(0), g\right)$ and that

$$
\lim _{i \rightarrow \infty} r_{0}\left(N_{i}\right)=\infty
$$

Also, suppose that $N_{i}$ separates infinity from the compact part. Then, there is a subsequence of $\left\{\tilde{N}_{i}\right\}$ which converges in Gromov-Hausdorff distance to a round 
sphere $S_{1}^{2}(a)$ of radius 1 and centered at a $\in \mathbb{R}^{3}$. Moreover, the convergence is $C^{2, \alpha}$ away from the origin.

Then, we use a smaller scale $r_{0}$ to blow down the surface

$$
\hat{N}=r_{0}(N)^{-1} N=\left\{r_{0}^{-1} x \mid x \in N\right\} .
$$

Lemma 3.2. Suppose that $\left\{N_{i}\right\}$ is a sequence of constant mean curvature surfaces in a given asymptotically flat end $\left(\mathbb{R}^{3} \backslash B_{1}(0), g\right)$ and that $\lim _{i \rightarrow \infty} r_{0}\left(N_{i}\right)=\infty$. Also, suppose that

$$
\lim _{i \rightarrow \infty} r_{0}\left(N_{i}\right) H\left(N_{i}\right)=0 .
$$

Then there is a subsequence of $\left\{\hat{N}_{i}\right\}$ converging to a 2-plane at distance 1 from the origin. Moreover the convergence is in $C^{2, \alpha}$ in any compact set of $\mathbb{R}^{3}$.

We must understand the behavior of the surfaces $N_{i}$ in the scales between $r_{0}\left(N_{i}\right)$ and $H^{-1}\left(N_{i}\right)$. We consider the scale $r_{i}$ such that

$$
\lim _{i \rightarrow \infty} \frac{r_{0}\left(N_{i}\right)}{r_{i}}=0, \quad \lim _{i \rightarrow \infty} r_{i} H\left(N_{i}\right)=0
$$

and blow down the surfaces

$$
\bar{N}_{i}=r_{i}^{-1} N=\left\{r_{i}^{-1} x \mid x \in N\right\} .
$$

Lemma 3.3. Suppose that $\left\{N_{i}\right\}$ is a sequence of constant mean curvature surfaces in a given asymptotically flat end $\left(\mathbb{R}^{3} \backslash B_{1}(0), g\right)$ and that

$$
\lim _{i \rightarrow \infty} r_{0}\left(N_{i}\right)=\infty \text {. }
$$

Also, suppose that $r_{i}$ are such that

$$
\lim _{i \rightarrow \infty} \frac{r_{0}\left(N_{i}\right)}{r_{i}}=0, \quad \lim _{i \rightarrow \infty} r_{i} H\left(N_{i}\right)=0 .
$$

Then there is a subsequence of $\left\{\bar{N}_{i}\right\}$ converging to a 2-plane at the origin in Gromov-Hausdorff distance. Moreover the convergence is $C^{2, \alpha}$ in any compact subset away from the origin.

\section{Asymptotic analysis}

In this section and the next two we assume that $(M, g, K)$ is an $(m, k, \varepsilon)$-AF-RT manifold, with $q=1$. First we revise [Qing and Tian 1997, Proposition 2.1], proving a different version. Set

$$
\|u\|_{1, i}^{2}=\int_{[(i-1) L, i L] \times S^{1}}|u|^{2}+|\nabla u|^{2} d t d \theta,
$$

where $(t, \theta)$ is the standard column coordinate. 
Lemma 4.1. Suppose $u \in W^{1,2}\left(\Sigma, \mathbb{R}^{k}\right)$ satisfies

$$
\Delta u+A \cdot \nabla u+B \cdot u=h
$$

in $\Sigma$, where $\Sigma=[0,3 L] \times S^{1}$ for L large. Then there exists a positive number $\delta_{0}$ such that if

$$
\|h\|_{L^{2}(\Sigma)} \leq \delta_{0} \max _{1 \leq i \leq 3}\|u\|_{1, i} \quad \text { and } \quad\|A\|_{L^{\infty}(\Sigma)} \leq \delta_{0}\|B\|_{L^{\infty}(\Sigma)} \leq \delta_{0},
$$

the following conditions are satisfied:

(a) $\|u\|_{1,3} \leq e^{-(1 / 2) L}\|u\|_{1,2}$ implies $\|u\|_{1,2}<e^{-(1 / 2) L}\|u\|_{1,1}$.

(b) $\|u\|_{1,1} \leq e^{-(1 / 2) L}\|u\|_{1,2}$ implies $\|u\|_{1,2}<e^{-(1 / 2) L}\|u\|_{1,3}$.

(c) If both

$$
\int_{L \times S^{1}} u d \theta \quad \text { and } \quad \int_{2 L \times S^{1}} u d \theta \leq \delta_{0} \max _{1 \leq i \leq 3}\|u\|_{1, i}
$$

then either $\|u\|_{1,2}<e^{-(1 / 2) L}\|u\|_{1,1}$ or $\|u\|_{1,2}<e^{-(1 / 2) L}\|u\|_{1,3}$.

Proof. Supposing $u \in W^{1,2}(\Sigma)$ and $u$ is harmonic, we can deduce that $u$ satisfies (a), (b) and this variant condition:

$\left(c^{\prime}\right)$ If both

$$
\int_{L \times S^{1}} u d \theta \text { and } \int_{2 L \times S^{1}} u d \theta=0
$$

then either $\|u\|_{1,2}<e^{-(1 / 2) L}\|u\|_{1,1}$ or $\|u\|_{1,2}<e^{-(1 / 2) L}\|u\|_{1,3}$.

A harmonic function $u$ can be written as

$$
u=a_{0}+b_{0} t+\sum_{n=1}^{\infty}\left(e^{n t}\left(a_{n} \cos n \theta+b_{n} \sin n \theta\right)+e^{-n t}\left(a_{-n} \cos n \theta+b_{-n} \sin n \theta\right)\right) .
$$

Then it follows that for $i=1,2,3$,

$$
\begin{aligned}
\|u\|_{1, i}^{2}=2 \pi & \left(\left(a_{0}^{2}+b_{0}^{2}\right) L+a_{0} b_{0} L^{2}(2 i-1)+\frac{1}{3} b_{0}^{2} L^{3}\left(3 i^{2}-3 i+1\right)\right) \\
+ & \frac{\pi}{2} \sum_{n=1}^{\infty}\left(\frac{e^{2 n L-1}}{n}\left(e^{2(i-1) n L}\left(a_{n}^{2}+b_{n}^{2}\right)+e^{-2 n i L}\left(a_{-n}^{2}+b_{-n}^{2}\right)\right)\right. \\
& \left.+4 L\left(a_{n} a_{-n}+b_{n} b_{-n}\right)\right) \\
+ & \pi \sum_{n=1}^{\infty}\left(\frac{e^{2 n L-1}}{n}\left(e^{2(i-1) n L}\left(n^{2} a_{n}^{2}+n^{2} b_{n}^{2}\right)+e^{-2 n i L}\left(n^{2} a_{-n}^{2}+n^{2} b_{-n}^{2}\right)\right)\right. \\
& \left.+4 L\left(n^{2} a_{n} a_{-n}+n^{2} b_{n} b_{-n}\right)\right) .
\end{aligned}
$$

If $L$ is fixed and sufficiently large, then

$$
\|u\|_{1,2}^{2}<\frac{1}{2}\left(e^{L}\|u\|_{1,3}^{2}+e^{-L}\|u\|_{1,1}^{2}\right),
$$


which implies (a). We get (b) in the same way. For $\left(\mathrm{c}^{\prime}\right)$, we have $a_{0}=b_{0}=0$, so then

$$
\|u\|_{1,2}^{2}<\frac{1}{2} e^{-L}\left(\|u\|_{1,3}^{2}+\|u\|_{1,1}^{2}\right)
$$

which implies $\left(\mathrm{c}^{\prime}\right)$.

The second step is to pass to limits. If the proposition were false, then one would have a sequence $\delta_{k} \rightarrow 0$ and a sequence of solutions $u_{k}$, each violating (a), (b), or (c), with $\left\|h_{k}\right\|_{L^{2}} \leq \delta_{k} \max _{1 \leq i \leq 3}\left\|u_{k}\right\|_{1, i},\left\|A_{k}\right\|_{\infty} \leq \delta_{k}$ and $\left\|B_{k}\right\|_{\infty} \leq \delta_{k}$ solving

$$
\Delta u_{k}+A_{k} \cdot \nabla u_{k}+B_{k} \cdot u_{k}=h_{k} .
$$

We may assume $\max _{1 \leq i \leq 3}\left\|u_{k}\right\|_{1, i}=1$; otherwise we can normalize them. So we know $\left\|u_{k}\right\|_{1,2}>C>0$, because $u_{k}$ violates (a), (b), or (c). We know that there is a subsequence that converges to some harmonic function $u \in W^{1,2}(\Sigma)$ weakly. From the interior $W^{2, p}$ estimate we know the convergence is strongly $W^{1,2}$ in $I_{2}$, which implies that $u$ is not trivially zero.

Because $u_{i} \rightarrow u$ weakly in $W^{1,2}(\Sigma)$ sense, we know $u_{i} \rightarrow u$ in the $W^{1,2}\left(I_{1}\right)$ and $W^{1,2}\left(I_{3}\right)$ senses. Then

$$
\liminf _{i \rightarrow \infty}\left\|u_{i}\right\|_{1,1} \geq\|u\|_{1,1}, \quad \lim _{i \rightarrow \infty}\left\|u_{i}\right\|_{1,2}=\|u\|_{1,2}, \quad \liminf _{i \rightarrow \infty}\left\|u_{i}\right\|_{1,3} \geq\|u\|_{1,3} .
$$

Then $u_{i}$ converges to some nontrivial harmonic function $u$ which violates one of (a), (b), or $\left(c^{\prime}\right)$, proving the lemma.

Given a surface $N$ in $\mathbb{R}^{3}$, recall from, for example, [Kenmotsu 2003, (8.5)], that

$$
\Delta_{e} v+\left|\nabla_{e} v\right|^{2} v=\nabla_{e} H_{e},
$$

where $v$ is the Gauss map from $N \rightarrow S^{2}$.

Lemma 4.2. For the constant mean curvature surfaces in the asymptotically flat end $\left(\mathbb{R}^{3} \backslash B_{1}(0), g\right)$, we have

$$
\left|\nabla_{e} H_{e}\right|(x) \leq C|x|^{-2} r_{0}^{-1} .
$$

Proof. Because the metric $g$ and the Euclidean metric are uniformly equivalent, we must prove that

$$
\left|\nabla H_{e}\right|(x) \leq C|x|^{-2} r_{0}^{-1} .
$$

From (2-10), (2-13), (2-14), and Lemma 2.5 (now $q=1$ ), we know that

$$
\begin{array}{rlrl}
\left|\nabla H_{e}\right| \leq\left|\bar{\nabla} h_{i j}\right||A|+\left|h_{i j}\right||A|^{2}+\left|h_{i j}\right|\left|\nabla \AA_{i j}\right|+H|A|\left|h_{i j}\right| & +H\left|\bar{\nabla} h_{i j}\right| \\
& +|A|\left|\bar{\nabla} h_{i j}\right|+\left|\bar{\nabla}^{2} h\right| \\
& \leq|x|^{-2} r_{0}^{-1}, &
\end{array}
$$

which completes the proof. 
Suppose $\Sigma$ is a constant mean curvature surface in the asymptotically flat end. Set

$$
A_{r_{1}, r_{2}}=\left\{x \in \Sigma\left|r_{1} \leq\right| x \mid \leq r_{2}\right\}
$$

and let $A_{r_{1}, r_{2}}^{0}$ be the standard annulus in $\mathbb{R}^{2}$. We are concerned with the behavior of $v$ on $A_{K r_{0}(\Sigma), s H^{-1}(\Sigma)}$ of $\Sigma$ where $K$ is fixed large and $s$ is fixed small. The lemma below gives us good coordinates on the surface.

Lemma 4.3. Suppose $\Sigma$ is a constant mean curvature surface in a given asymptotically flat end $\left(\mathbb{R}^{3} \backslash B_{1}(0), g\right)$. Then, for any $\varepsilon>0$ and L large, there are $M, s$ and $K$ such that, if $r_{0} \geq M$ and $K r_{0}(\Sigma)<r<s H^{-1}(\Sigma)$, then $\left(r^{-1} A_{r, e^{L} r}, r^{-2} g_{e}\right)$ may be represented as $\left(A_{1, e^{L}}^{0}, \bar{g}\right)$ and

$$
\left\|\bar{g}-|d x|^{2}\right\|_{C^{1}\left(A_{\left.1, e^{L}\right)}^{0}\right.} \leq \varepsilon .
$$

In other words, in the cylindrical coordinates $\left(S^{1} \times\left[\log r, L+\log r, \bar{g}_{c}\right]\right)$,

$$
\left\|\bar{g}_{c}-\left(d t^{2}+d \theta^{2}\right)\right\|_{C^{1}\left(S^{1} \times[\log r, L+\log r]\right)} \leq \varepsilon .
$$

Proof. Suppose this is not true. Then we can assume that such $K$ (or such $s$ ) cannot be found. Then by Lemma 3.2, for some $\varepsilon_{0}>0$, there is a sequence $\Sigma_{n}$ with $r_{0}\left(\Sigma_{n}\right) \rightarrow \infty$ and $\tilde{l}_{n} \rightarrow \infty$ such that

$$
\left(\left(K r_{0} e^{\tilde{l}_{n} L}\right)^{-1} A_{K r_{0} e^{\tilde{l}_{n} L}, K r_{0} e^{\left.\tilde{l}_{n}+1\right) L}},\left(K r_{0} e^{\tilde{l}_{n} L}\right)^{-2} g_{e}\right)
$$

is not $\varepsilon_{0}$ close to $\left(A_{1, e^{L}}^{0}, \bar{g}\right)$.

By Lemma 3.1,

$$
\frac{K r_{0} e^{\tilde{l}_{n} L}}{s H^{-1}\left(\Sigma_{n}\right)} \rightarrow 0
$$

must hold because we have chosen $s$ sufficiently small.

So if we assume $r_{n}=K r_{0} e^{\tilde{l}_{n} L}$, then

$$
\lim _{n \rightarrow \infty} \frac{r_{n}}{K r_{0}}=\infty, \quad \lim _{n \rightarrow \infty} \frac{r_{n}}{s H^{-1}}=0 .
$$

Blowing down the surface using $r_{n}$ gives a contradiction with Lemma 3.3.

Now consider the cylindrical coordinates $(t, \theta)$ on $\left(S^{1} \times\left[\log K r_{0}, \log s H^{-1}\right]\right)$. The tension field satisfies

$$
|\tau(v)|=r^{2}\left|\nabla_{e} H_{e}\right| \leq C r_{0}^{-1}
$$

for $t \in\left[\log K r_{0}, \log s H^{-1}\right]$. Thus,

$$
\int_{S^{1} \times[t, t+L]}|\tau(v)|^{2} d t d \theta \leq C r_{0}^{-2} .
$$

Let $I_{i}$ be $S^{1} \times\left[\log K r_{0}+(i-1) L, \log K r_{0}+i L\right]$, and $N_{i}$ be $I_{i-1} \cup I_{i} \cup I_{i+1}$. On $\Sigma_{n}$, assume $\log \left(s H^{-1}\right)-\log \left(K r_{0}\right)=l_{n} L$. As in [Qing and Tian 1997], first we prove: 
Lemma 4.4. For each $i \in\left[3, l_{n}-2\right]$, there exists a geodesic $\gamma$ such that

$$
\int_{I_{i}}|\widetilde{\nabla}(v-\gamma)|^{2} d t d \theta \leq C\left(e^{-i L}+e^{-\left(l_{n}-i\right) L}\right) s^{2}+C r_{0}^{-1}
$$

where $\widetilde{\nabla}$ is the gradient on $S^{1} \times\left[\log \left(K r_{0}\right), \log \left(s H^{-1}\right)\right]$.

Lemma 4.5. By Theorem 2.7,

$$
[v]_{C^{\alpha}\left(I_{i}\right)} \leq\|\widetilde{\nabla} v\|_{L^{\infty}} \leq C\left(r_{0}^{-1 / 2}+s\right) .
$$

Thus if $r_{0}$ sufficiently large and s sufficiently small, then $[v]_{C^{\alpha}\left(N_{i}\right)}$ is very small.

Proof of Lemma 4.4. To apply the Lemma 4.1 to prove this lemma we choose points $P$ and $Q$ on $S^{2}$ (the image of Gauss map) satisfying

$$
\begin{gathered}
\left|P-\frac{1}{2 \pi} \int_{(i-1) L \times S^{1}} v d \theta\right| \leq C \max _{(i-1) L \times S^{1}}|v-P|^{2}, \\
\left|Q-\frac{1}{2 \pi} \int_{i L \times S^{1}} v d \theta\right| \leq C \max _{i L \times S^{1}}|v-Q|^{2} .
\end{gathered}
$$

Note that $S^{2}$ is compact and smooth, so by Lemma 4.5 we can always find such $P$ and $Q$ that are very close. So there is a unique geodesic $\gamma_{i}$ connecting $P$ and $Q$ whose velocity is sufficiently small.

If we write down the equation satisfied by $v-\gamma_{i}$ on $S^{1} \times\left[\log \left(K r_{0}\right), \log \left(s H^{-1}\right)\right]$,

$$
\tilde{\Delta} u+A \cdot \widetilde{\nabla} u+B \cdot u=\tau,
$$

where $u=v-\gamma_{i}$, we have

$$
\begin{aligned}
& |A| \leq C\left(|\widetilde{\nabla} v|+\left|\widetilde{\nabla} \gamma_{i}\right|\right) \leq \delta_{0}, \\
& |B| \leq C \min \left\{|\widetilde{\nabla} v|^{2},\left|\widetilde{\nabla} \gamma_{i}\right|^{2}\right\} \leq \delta_{0} .
\end{aligned}
$$

If Lemma 4.1(c) cannot be used, the only reason is that

$$
\left\|v-\gamma_{i}\right\|_{1, i} \leq C\|\tau\|_{L^{2}\left(N_{i}\right)},
$$

and so

$$
\int_{I_{i}}\left|\widetilde{\nabla}\left(v-\gamma_{i}\right)\right|^{2} d t d \theta \leq C r_{0}^{-2}
$$

which implies (4-1).

If Lemma 4.1(c) can be used, then applying it for $u=v-\gamma_{i}$ over $N_{i}$, we have one of the following:

$$
\begin{aligned}
& \|u\|_{1, i}<e^{-(1 / 2) L}\|u\|_{1, i-1}, \\
& \|u\|_{1, i}<e^{-(1 / 2) L}\|u\|_{1, i+1} .
\end{aligned}
$$


Suppose the first one happens (without loss of generality). Then we may push this relation to the left because (4-2) holds regardless of $t$ 's position. If the theorem can be used on $N_{j+1}$ but not on $N_{j}$ for some $j \geq 2$, then

$$
\|u\|_{1, i}<e^{-(1 / 2)(i-j) L}\|u\|_{1, j} \leq C e^{-(1 / 2)(i-j) L} r_{0}^{-1} \leq C r_{0}^{-1} .
$$

If the theorem can be used until $I_{2}$, then

$e^{L / 2}\|u\|_{1,2} \leq\|u\|_{1,1}$

$$
\begin{aligned}
&=\left(\int_{I_{1}} u^{2} d t d \theta\right)^{1 / 2}+\left(\int_{I_{1}}|\widetilde{\nabla} u|^{2} d t d \theta\right)^{1 / 2} \\
& \leq\left(\int_{I_{2}} u^{2} d t d \theta\right)^{1 / 2}+\left(\int_{I_{1}}(u(t, \theta)-u(t+L, \theta))^{2} d t d \theta\right)^{1 / 2} \\
&+\left(\int_{I_{1}}|\widetilde{\nabla} u|^{2} d t d \theta\right)^{1 / 2} .
\end{aligned}
$$

So we have

$$
\begin{aligned}
\left(e^{L / 2}-1\right)\|u\|_{1,2} & \leq\left(\int_{I_{1}}\left(\int_{0}^{L}\left|\frac{\partial u}{\partial t}(t+s, \theta)\right| d s\right)^{2} d t d \theta\right)^{1 / 2}+\left(\int_{I_{1}}|\widetilde{\nabla} u|^{2} d t d \theta\right)^{1 / 2} \\
& \leq \int_{0}^{L}\left(\int_{I_{1}}\left|\frac{\partial u}{\partial t}(t+s, \theta)\right|^{2} d t d \theta\right)^{1 / 2} d s+\left(\int_{I_{1}}|\widetilde{\nabla} u|^{2} d t d \theta\right)^{1 / 2} \\
& \leq C\left(\int_{I_{1} \cup I_{2}}|\widetilde{\nabla} u|^{2} d t d \theta\right)^{1 / 2} \\
& \leq C\left(\int_{I_{1} \cup I_{2}}|\widetilde{\nabla} v|^{2} d t d \theta\right)^{1 / 2}+C\left(\int_{I_{1} \cup I_{2}}\left|\widetilde{\nabla} \gamma_{i}\right|^{2} d t d \theta\right)^{1 / 2} \\
& \leq C\left(r_{0}^{-1 / 2}+s\right) .
\end{aligned}
$$

This implies the estimate

$$
\|u\|_{1, i} \leq C e^{(-(i-2) / 2) L}\|u\|_{1,2} \leq C e^{-(i / 2) L}\left(r_{0}^{-1 / 2}+s\right) .
$$

If $\|u\|_{1, i}<e^{-(1 / 2) L}\|u\|_{1, i+1}$ happens, we will have similarly

$$
\|u\|_{1, i} \leq C e^{-\left(\left(l_{n}-i\right) / 2\right) L}\left(r_{0}^{-1 / 2}+s\right) .
$$

Finally we get

$$
\|u\|_{1, i} \leq C\left(e^{-(i / 2) L}+e^{-\left(\left(l_{n}-i\right) / 2\right) L}\right) s+C r_{0}^{-1 / 2},
$$

which implies (4-1).

Then to get the energy decay, we use the Hopf differential

$$
\Phi=\left|\partial_{t} v\right|^{2}-\left|\partial_{\theta} v\right|^{2}-2 \sqrt{-1} \partial_{t} v \cdot \partial_{\theta} v .
$$


We know that the $L^{1}$ norm of $\Phi$ is invariant under conformal change of the co-

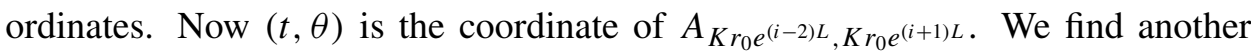
coordinate for it: Set $r_{i}=K r_{0} e^{i L}$. Then

$$
\left(r_{i}^{-1} A_{\left.K r_{0} e^{(i-2) L}, K r_{0} e^{(i+1) L}, r_{i}^{-2} g_{e}\right)}\right.
$$

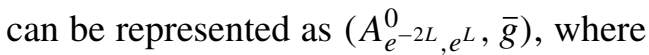

$$
\left\|\bar{g}-|d x|^{2}\right\|_{C^{1}\left(A_{e}^{0}-2 L, e^{L}\right)} \leq \varepsilon .
$$

Assume this Euclidean coordinate is $(x, y)$, so

$$
\int_{S^{1} \times\left[\log K r_{0}+(i-1) L, \log K r_{0}+i L\right]}|\Phi| d t d \theta=\int_{A_{e}^{0}-L, 1}|\Phi| d x d y .
$$

To estimate the right hand side, we use the Cauchy integral formula on $\Omega=$ $A_{e^{-2 L}, e^{L}}^{0}$, and set $\Omega^{\prime}=A_{e^{-L}, 1}^{0}$. Then for any $z \in \Omega^{\prime}$,

$$
\Phi(v)(z)=\frac{1}{2 \pi \sqrt{-1}} \int_{\partial \Omega} \frac{\Phi(w)}{w-z} d w+\frac{1}{2 \pi \sqrt{-1}} \int_{\Omega} \frac{\partial \Phi(w)}{\partial \bar{w}} \frac{d w \wedge d \bar{w}}{w-z} .
$$

We know

$$
\begin{aligned}
\left|\partial_{x} v\right|,\left|\partial_{y} v\right| \leq C K r_{0} e^{i L}|A| & \leq C K r_{0} e^{i L}\left(|x|^{-1} r_{0}^{-1 / 2}+r_{1}^{-1}\right) \\
& \leq C\left(r_{0}^{-1 / 2}+s e^{-\left(l_{n}-i\right) L}\right),
\end{aligned}
$$

so we have

$$
\left|\frac{1}{2 \pi \sqrt{-1}} \int_{\partial \Omega} \frac{\Phi(w)}{w-z} d w\right| \leq C\left(r_{0}^{-1}+s^{2} e^{-2\left(l_{n}-i\right) L}\right) .
$$

For the second term, notice that by easy calculation

$$
\frac{\partial \Phi(w)}{\partial \bar{w}}=\partial v \cdot \bar{\tau}(v)
$$

where $\bar{\tau}(v)$ is the tension field under this coordinate. Now,

$$
|\bar{\tau}(v)| \leq\left(K r_{0} e^{i l}\right)^{2}\left|\nabla_{e} H_{e}\right| \leq C r_{0}^{-1}
$$

so we have

Then we get

$$
\frac{1}{2 \pi \sqrt{-1}} \int_{\Omega} \frac{\partial \Phi(w)}{\partial \bar{w}} \frac{d w \wedge d \bar{w}}{w-z} \leq C r_{0}^{-1}
$$

$$
\int_{\Omega^{\prime}}|\Phi| \leq C\left(r_{0}^{-1}+s^{2} e^{-2\left(l_{n}-i\right) L}\right) .
$$


By direct calculation,

$$
\begin{aligned}
& \int_{S^{1} \times\left[K r_{0} e^{(i-1) L}, K r_{0} e^{i L}\right]}\left|\partial_{t} v\right|^{2} d t d \theta \\
& \quad \leq \int_{S^{1} \times\left[K r_{0} e^{(i-1) L}, K r_{0} e^{i L}\right]}|\Phi| d t d \theta+\int_{S^{1} \times\left[K r_{0} e^{(i-1) L}, K r_{0} e^{i L}\right]}\left|\partial_{\theta} v\right|^{2} d t d \theta,
\end{aligned}
$$

and we can get the estimate of

$$
\int_{S^{1} \times\left[K r_{0} e^{(i-1) L}, K r_{0} e^{i L}\right]}\left|\partial_{\theta} v\right|^{2} d t d \theta
$$

directly by (4-1). So we get the estimate

$$
\int_{S^{1} \times\left[K r_{0} e^{(i-1) L}, K r_{0} e^{i L}\right]}|\widetilde{\nabla} v|^{2} d t d \theta \leq C\left(e^{-i L}+e^{-\left(l_{n}-i\right) L}\right) s^{2}+C r_{0}^{-1} .
$$

Proposition 4.6. Suppose that $\left\{\Sigma_{n}\right\}$ is a sequence of constant mean curvature surfaces in a given asymptotically flat end $\left(\mathbb{R}^{3} \backslash B_{1}(0), g\right)$ and that

$$
\lim _{i \rightarrow \infty} r_{0}\left(\Sigma_{n}\right)=\infty
$$

Also, suppose that

$$
\lim _{n \rightarrow \infty} r_{0}\left(\Sigma_{n}\right) H\left(\Sigma_{n}\right)=0 .
$$

Then there exists a large number $K$, a small number $s$ and a number $n_{0}$ such that, when $n \geq n_{0}$,

$$
\max _{I_{i}}|\tilde{\nabla} v| \leq C\left(e^{-(i / 2) L}+e^{-\left(\left(l_{n}-i\right) / 2\right) L}\right) s+C r_{0}^{-1 / 2},
$$

where

$$
\begin{gathered}
I_{i}=S^{1} \times\left[\log \left(K r_{0}\left(\Sigma_{n}\right)\right)+(i-1) L, \log \left(K r_{0}\left(\Sigma_{n}\right)\right)+i L\right], \\
i \in\left[0, l_{n}\right] \log \left(K r_{0}\left(\Sigma_{n}\right)\right)+l_{n} L=\log \left(s H^{-1}\left(\Sigma_{n}\right)\right) .
\end{gathered}
$$

Proof. We use the interior estimate of the elliptic equation

$$
\tilde{\Delta} v+|\widetilde{\nabla} v|^{2} v=\tau
$$

We know $\|\widetilde{\nabla} v\|_{\infty} \leq C\left(r_{0}^{-1 / 2}+s\right)$, and $\|\tau\|_{\infty} \leq C r_{0}^{-1}$. Assume that

$$
I_{i} \subset \subset \tilde{I}_{i} \subset \subset N_{i} .
$$

Then for some $p>2$,

$$
\begin{aligned}
\sup _{I_{i}}|\widetilde{\nabla} v| & \leq C\|\widetilde{\nabla} v\|_{W^{1, p}\left(I_{i}\right)} \leq C\left(\|v\|_{L^{p}\left(\tilde{I}_{i}\right)}+r_{0}^{-1}\right) \leq C\left(\|v\|_{L^{2}\left(N_{i}\right)}+r_{0}^{-1}\right) \\
& \leq C\left(e^{-(i / 2) L}+e^{-\left(\left(l_{n}-i\right) / 2\right) L}\right) s+C r_{0}^{-1 / 2} .
\end{aligned}
$$


This analysis improves our understanding of the blowdowns that we discussed in the previous section. Namely,

Corollary 4.7. Assume the same conditions as in Proposition 4.6 and, in addition,

$$
\lim _{r_{0} \rightarrow \infty} \frac{\log r_{1}}{r_{0}^{1 / 4}}=0
$$

Then the limit plane in Lemmas 3.2 and 3.3 are all orthogonal to the same vector a. In fact, we may choose s small and i large enough so that

$$
|v(x)+a| \leq \varepsilon
$$

for all $x \in \Sigma_{n}$ and $|x| \leq s H^{-1}\left(\Sigma_{n}\right)$.

Proof. We want to prove that

$$
\operatorname{Osc}_{B_{s H^{-1}} \cap \Sigma_{n}} v
$$

is sufficiently small if $r_{0}\left(\Sigma_{n}\right)$ is large and $s$ is small. We already know that

$$
\operatorname{Osc}_{B_{K r_{0}} \cap \Sigma_{n}} v
$$

is very small from Lemma 3.2, so we need only to prove that

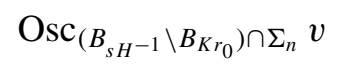

is small.

From Proposition 4.6 above we find that

$$
\begin{aligned}
\operatorname{Osc}_{\left(B_{s H^{-1}} \backslash B_{K r_{0}}\right) \cap \Sigma_{n}} v & \leq \sum_{i=1}^{l_{n}} \operatorname{Osc}_{I_{i}} v \leq C \sum_{i=1}^{l_{n}} \sup _{I_{i}}|\widetilde{\nabla} v| \\
& \leq C \sum_{i=1}^{l_{n}}\left(\left(e^{-(i / 2) L}+e^{-\left(\left(l_{n}-i\right) / 2\right) L}\right) s+r_{0}^{-1 / 2}\right) \leq C s+l_{n} r_{0}^{-1 / 2} .
\end{aligned}
$$

From the inequalities $C^{-1} r_{1} \leq H^{-1} \leq C r_{1}$, we have

$$
l_{n} r_{0}^{-1 / 2}=L^{-1}\left(\log \left(s H^{-1}\right)-\log \left(K r_{0}\right)\right) r_{0}^{-1 / 2} \leq C \frac{\log r_{1}}{r_{0}^{1 / 2}} \rightarrow 0
$$

as $r_{0} \rightarrow \infty$, which proves the corollary. 
Corollary 4.8. Assume the same conditions as in Proposition 4.6. Let $v_{n}=v\left(p_{n}\right)$ for some $p_{n} \in I_{l_{n} / 2}$. Then for $i \in\left[0, \frac{1}{2} l_{n}\right]$,

$$
\sup _{I_{i}}\left|v-v_{n}\right| \leq C\left(e^{-(1 / 2) i L}+e^{-(1 / 4) l_{n} L}\right) s+l_{n} r_{0}^{-1 / 2},
$$

and for $i \in\left[\frac{1}{2} l_{n}, l_{n}\right]$,

$$
\sup _{I_{i}}\left|v-v_{n}\right| \leq C\left(e^{-(1 / 4) l_{n} L}+e^{-(1 / 2)\left(l_{n}-i\right) L}\right) s+l_{n} r_{0}^{-1 / 2} .
$$

\section{Harmonic coordinates}

We assume that the metric $g$ can be expanded in the coordinate $\left\{x^{i}\right\}$ as

$$
g_{i j}=\delta_{i j}+h_{i j}=\delta_{i j}+h_{i j}^{1}(\theta) / r+Q,
$$

where $\theta$ is the coordinate on the unit sphere $S^{2}, h_{i j}^{1}(\theta)$ is a function extended constantly along the radial direction, and $Q$ satisfies

$$
\sup r^{2+k}\left|\partial^{k} Q\right| \leq C
$$

for $k=0,1, \ldots, 5$.

First, note that

$$
\begin{aligned}
\Delta_{g} x^{k} & =\frac{1}{\sqrt{g}} \frac{\partial}{\partial x^{i}}\left(\sqrt{g} g^{i j} \frac{\partial}{\partial x^{j}} x^{k}\right) \\
& =\frac{\partial}{\partial x^{i}} g^{i k}+\frac{1}{2} g^{i k} g^{m n} g_{m n, i}=-g^{m n} \Gamma_{m n}^{k}=O\left(|x|^{-2}\right) .
\end{aligned}
$$

Our aim is to find an asymptotically harmonic coordinate, that is, a coordinate $y^{i}$ such that $\Delta_{g} y^{k}=O\left(|x|^{-3}\right)$ :

$$
\begin{aligned}
\Delta_{g} x^{k} & =-g^{j l} g^{i k} \frac{1}{2}\left(\frac{\partial}{\partial x^{j}} h_{l i}+\frac{\partial}{\partial x^{l}} h_{j i}-\frac{\partial}{\partial x^{i}} h_{j l}\right) \\
& =-g^{j l} g^{i k} \frac{1}{2}\left(r ^ { - 2 } \left(\left(h_{l i, j}^{1}(\theta)-h_{l i}^{1}(\theta) \frac{x^{j}}{r}\right)+\left(h_{j i, l}^{1}(\theta)-h_{j i}^{1}(\theta) \frac{x^{l}}{r}\right)\right.\right. \\
& =-g^{j l} g^{i k} \frac{1}{2} r^{-2} f_{l i j}^{1}(\theta)+O\left(|x|^{-3}\right) .
\end{aligned}
$$

We also know that $g^{i j}=\delta^{i j}-h_{i j}^{1}(\theta) / r+O\left(r^{-2}\right)$.

Then

$$
\Delta_{g} x^{k}=-\frac{1}{2} r^{-2} f_{j k j}^{1}(\theta)+O\left(r^{-3}\right) .
$$

Suppose $0=\xi_{0}>\xi_{1} \geq \xi_{2} \geq \cdots$ are the eigenvalues of $\left.\Delta\right|_{S^{2}}$, and $A_{n}(\theta)$ are the corresponding orthonormal eigenvectors. 
Set

We have

$$
y^{k}=x^{k}+\sum_{n=0}^{\infty} f_{n}^{k}(r) A_{n}(\theta)
$$

$$
\Delta_{g} y^{k}=\Delta_{g} x^{k}+\sum_{n=0}^{\infty} \Delta_{\mathbb{R}^{3}}\left(f_{n}^{k}(r) A_{n}(\theta)\right)+\sum_{n=0}^{\infty}\left(\Delta_{g}-\Delta_{\mathbb{R}^{3}}\right)\left(f_{n}^{k}(r) A_{n}(\theta)\right) .
$$

Solve the equation

$$
\Delta_{g} x^{k}+\sum_{n=0}^{\infty} \Delta_{\mathbb{R}^{3}}\left(f_{n}^{k}(r) A_{n}(\theta)\right)=O\left(|x|^{-3}\right) .
$$

Assume

So we have

$$
\frac{1}{2} f_{j k j}^{1}(\theta)=\sum_{n=0}^{\infty} \lambda_{n}^{k} A_{n}(\theta)
$$

$$
\begin{array}{rlrl}
\sum_{n=0}^{\infty} \Delta_{\mathbb{R}^{3}}\left(f_{n}^{k}(r) A_{n}(\theta)\right) & =r^{-2} \sum_{n=0}^{\infty} \lambda_{n}^{k} A_{n}(\theta), & \\
\frac{1}{r^{2}}\left(2 r f_{n}^{k \prime}+r^{2} f_{n}^{k \prime \prime}+f_{n}^{k}(r) \xi_{n}\right) & =\lambda_{n}^{k} & & n=0, \ldots, \infty, \\
f_{0}^{k} & =\lambda_{0}^{k} \log r, & & \\
f_{n}^{k} & =\frac{\lambda_{n}^{k}}{\xi_{n}} & & n>0,
\end{array}
$$

and this solution satisfies

$$
\sum_{n=0}^{\infty}\left(\Delta_{g}-\Delta_{\mathbb{R}^{3}}\right)\left(f_{n}^{k}(r) A_{n}(\theta)\right)=O\left(|x|^{-3}\right) .
$$

So if

$$
y^{k}=x^{k}+\frac{1}{2 \sqrt{\pi}} \lambda_{0}^{k} \log r+\sum_{n=1}^{\infty} \frac{\lambda_{n}^{k}}{\xi_{n}} A_{n}(\theta),
$$

then we must have

$$
\Delta y^{k}=O\left(|x|^{-3}\right) .
$$

Note that

$$
\left.\Delta\right|_{S^{2}} \sum_{n=1}^{\infty} \frac{\lambda_{n}^{k}}{\xi_{n}} A_{n}(\theta)=\sum_{n=1}^{\infty} \lambda_{n}^{k} A_{n}(\theta)=\frac{1}{2} f_{j k j}^{1}(\theta)-\frac{1}{2} \overline{f_{j k j}^{1}(\theta)}
$$

where $\overline{f_{j k j}^{1}(\theta)}$ is its mean value on the unit sphere. 
Set

$$
\begin{aligned}
g_{k}^{1}(\theta) & =\sum_{n=1}^{\infty} \frac{\lambda_{n}^{k}}{\xi_{n}} A_{n}(\theta)=\Delta^{-1}\left(\frac{1}{2} f_{j k j}^{1}(\theta)-\frac{1}{2} \overline{f_{j k j}^{1}(\theta)}\right), \\
\frac{\partial y^{k}}{\partial x^{i}} & =\delta_{i k}+\frac{\lambda_{0}^{k}}{2 \sqrt{\pi}} \frac{1}{r} \frac{x^{i}}{r}+g_{k}^{1}(\theta)_{i} \frac{1}{r} \\
\frac{\partial x^{i}}{\partial y^{k}} & =\delta_{i k}+O\left(|x|^{-1}\right) .
\end{aligned}
$$

So we get

$$
\tilde{g}_{i j}=g\left(\frac{\partial}{\partial y^{i}}, \frac{\partial}{\partial y^{j}}\right)=\delta_{i j}+O\left(|x|^{-1}\right) .
$$

We now define $\tilde{h}_{i j}$ by

$$
\tilde{g}_{i j}=\delta_{i j}+\tilde{h}_{i j}
$$

and discuss its ellipticity. We have

$$
\tilde{h}_{i j}=h_{i j}-\frac{1}{2 r \sqrt{\pi}}\left(\lambda_{0}^{i} \frac{x^{j}}{r}+\lambda_{0}^{j} \frac{x^{i}}{r}\right)-\frac{\left(g_{i, j}^{1}(\theta)+g_{j, i}^{1}(\theta)\right)}{r},
$$

where $g_{i, j}^{1}(\theta)$ denotes the constant extension along the radial direction of function $\left.\left(\partial g_{i}^{1}(\theta) / \partial x^{j}\right)\right|_{S^{2}}$.

Example 5.1. For the metric $g_{i j}=\delta_{i j}+\delta_{i j} / r$, we have

$$
\Delta_{g} x^{k}=-\frac{1}{2} \frac{x^{k}}{r^{3}}+O\left(|x|^{-3}\right) .
$$

On $S^{2}$, we have $\left.\Delta\right|_{S^{2}} x^{k}=-2 x^{k}$. So if we let

$$
y^{k}=x^{k}-\frac{1}{4} \frac{x^{k}}{r}
$$

then $\Delta_{g} y^{k}=O\left(|x|^{-3}\right)$. Thus,

$$
\begin{aligned}
\frac{\partial y^{k}}{\partial x^{i}} & =\delta_{k i}-\frac{1}{4}\left(\frac{\delta_{k i}}{r}-\frac{x^{k} x^{i}}{r^{3}}\right), \\
\tilde{h}_{i j} & =\frac{3 \delta_{i j}}{2 r}-\frac{x^{i} x^{j}}{2 r^{3}}+O\left(r^{-2}\right) .
\end{aligned}
$$

Lemma 5.2. Suppose in some coordinate $\left\{x^{i}\right\}$ that $g_{i j}=\delta_{i j}+h_{i j}^{1}(\theta) / r+Q$. Then for any $m>2$ there exists $\varepsilon>0$ such that if $\left\|h_{i j}^{1}(\theta)-\delta_{i j}(\theta)\right\|_{W^{m, 2}\left(S^{2}\right)} \leq \varepsilon$, then in the asymptotically harmonic coordinate $\left\{y^{i}\right\}$ from above, we have

$$
\tilde{g}_{i j}=\delta_{i j}+\tilde{h}_{i j}
$$

where $\tilde{h}_{i j}=O\left(|y|^{-1}\right) s$ and $|y| \tilde{h}_{i j}$ is uniformly elliptic. 
Proof. We know easily from (5-2) that $\tilde{h}_{i j}=O\left(|x|^{-1}\right)$ and that $\lim _{|x| \rightarrow \infty}|y| /|x|=1$. Then $\tilde{h}_{i j}=O\left(|y|^{-1}\right)$. So we need only to prove that $|y| \tilde{h}_{i j}$ is uniformly elliptic.

First, from $\left\|h_{i j}^{1}(\theta)-\delta_{i j}(\theta)\right\|_{W^{m, 2}\left(S^{2}\right)} \leq \varepsilon$,

$$
\left\|\frac{1}{2} f_{j k j}^{1}(\theta)-\frac{1}{2} \frac{x^{k}}{r}\right\|_{W^{m-1,2}\left(S^{2}\right)} \leq C \varepsilon .
$$

Note that $\frac{1}{2} f_{j k j}^{1}(\theta)=\sum_{n=0}^{\infty} \lambda_{n}^{k} A_{n}(\theta)$ and $x^{k}$ is an eigenvector of $\Delta_{S^{2}}$, so we can assume that $A_{1}(\theta)=\left.C_{k} x^{k}\right|_{S^{2}}$ without loss of generality. Now,

$$
\left\|\lambda_{0}^{k} A_{0}(\theta)+\left(\lambda_{1}^{k} C_{k}-\frac{1}{2}\right) x^{k}+\sum_{n=2}^{\infty} \lambda_{n}^{k} A_{n}(\theta)\right\|_{W^{m-1,2}\left(S^{2}\right)} \leq \varepsilon,
$$

so we get

$$
\left|\lambda_{0}^{k}\right| \leq \varepsilon, \quad \lambda_{1}^{k} C_{k}-\frac{1}{2} \leq \varepsilon, \quad \sum_{n=2}^{\infty}\left(\left|\xi_{n}\right|^{(m-1) / 2} \lambda_{n}^{k}\right)^{2} \leq \varepsilon .
$$

By $(5-1)$,

$$
\frac{\partial y^{k}}{\partial x^{i}}=\delta_{i k}+\frac{\lambda_{0}^{k}}{2 \sqrt{\pi}} \frac{1}{r} \frac{x^{i}}{r}-\frac{1}{2}\left(\frac{1}{2} \pm \varepsilon\right)\left(\frac{\delta_{i k}}{r}-\frac{x^{i} x^{k}}{r^{3}}\right)+\sum_{n=2}^{\infty} \frac{\lambda_{n}^{k}}{\xi_{n}} \frac{\partial A_{n}(\theta)}{\partial x^{i}},
$$

where the last term on the right can be estimated, for some $p>0$, as

$$
\begin{aligned}
\mid \sum_{n=2}^{\infty} \frac{\lambda_{n}^{k}}{\xi_{n}} \frac{\partial A_{n}(\theta)}{\partial x^{i} \mid} & \leq \sum_{n=2}^{\infty} \frac{\left|\lambda_{n}^{k}\right|}{\left|\xi_{n}\right|} \frac{\left|\nabla_{S^{2}} A_{n}(\theta)\right|}{r} \\
& \leq \sum_{n=2}^{\infty} \frac{\left|\lambda_{n}^{k}\right|}{\left|\xi_{n}\right|} \frac{\left\|A_{n}(\theta)\right\|_{W^{2+p, 2}}}{r} \\
& \leq \sum_{n=2}^{\infty} \frac{\left|\lambda_{n}^{k}\right|}{\left|\xi_{n}\right|} \frac{\left|\xi_{n}\right|^{1+p / 2}\left\|A_{n}(\theta)\right\|_{L^{2}}}{r} \\
& \leq \frac{1}{r} \sum_{n=2}^{\infty}\left|\lambda_{n}^{k}\right|\left|\xi_{n}\right|^{(m-1) / 2}\left|\xi_{n}\right|^{(p-m+1) / 2} \\
& \leq \frac{1}{r}\left(\sum_{n=2}^{\infty}\left(\left|\lambda_{n}^{k}\right|\left|\xi_{n}\right|^{(m-1) / 2}\right)^{2}\right)^{1 / 2}\left(\sum_{n=2}^{\infty}\left|\xi_{n}\right|^{p-m+1}\right)^{1 / 2} .
\end{aligned}
$$

Let $p=(m-2) / 2$. Then from $\xi_{n}=O(n)$ we have

$$
\sum_{n=2}^{\infty}\left|\xi_{n}\right|^{p-m+1} \leq C
$$


so

Then we have

$$
\left|\sum_{n=2}^{\infty} \frac{\lambda_{n}^{k}}{\xi_{n}} \frac{\partial A_{n}(\theta)}{\partial x^{i}}\right| \leq \frac{C \varepsilon}{r} .
$$

$$
\frac{\partial y^{k}}{\partial x^{i}}=\delta_{i k}-\frac{1}{4}\left(\frac{\delta_{i k}}{r}-\frac{x^{i} x^{k}}{r^{3}}\right)+\frac{C \varepsilon}{r},
$$

so we can deduce that

$$
\tilde{h}_{i j}=h_{i j}+\frac{\delta_{i j}}{2 r}-\frac{x^{i} x^{j}}{2 r^{3}}+\frac{C \varepsilon}{r} .
$$

It follows from $\left\|h_{i j}^{1}(\theta)-\delta_{i j}(\theta)\right\|_{W^{m, 2}\left(S^{2}\right)} \leq \varepsilon$ that $r h_{i j}$ is uniformly elliptic. The eigenvalues of $\left(x^{i} x^{j}\right) / r^{2}$ are between 0 and 1 , so $|y| \tilde{h}_{i j}$ is uniformly elliptic, from the fact that $\lim _{r \rightarrow \infty}|y| / r=1$ and $\varepsilon$ is sufficiently small.

So all the analysis in Sections 2-4 can be done in the asymptotically harmonic coordinate $\left\{y^{i}\right\}$.

Lemma 5.3. In the asymptotically harmonic coordinate $\left\{y^{i}\right\}$, we have

$$
-\frac{1}{2} \Delta_{g} \log |\tilde{g}|=R(g)+O\left(|y|^{-4}\right) .
$$

Proof. From direct calculation we have

$$
\begin{aligned}
R(g) & =\tilde{g}^{j k} \tilde{g}^{i l} \tilde{g}_{m l}\left(\frac{\partial \widetilde{\Gamma}_{j k}^{m}}{\partial y^{i}}-\frac{\partial \widetilde{\Gamma}_{i k}^{m}}{\partial y^{j}}\right)+O\left(|y|^{-4}\right), \\
\tilde{g}^{j k} \tilde{g}^{i l} \tilde{g}_{m l} \frac{\partial \widetilde{\Gamma}_{j k}^{m}}{\partial y^{i}} & =\tilde{g}^{i l} \tilde{g}_{m l} \frac{\partial\left(\tilde{g}{ }^{j k} \widetilde{\Gamma}_{j k}^{m}\right)}{\partial y^{i}}+O\left(|y|^{-4}\right) \\
& =-\tilde{g}^{i l} \tilde{g}_{m l} \frac{\partial \Delta_{g} y^{m}}{\partial y^{i}}+O\left(|y|^{-4}\right)=O\left(|y|^{-4}\right), \\
-\tilde{g}^{j k} \tilde{g}^{i l} \tilde{g}_{m l} \frac{\partial \widetilde{\Gamma}_{i k}^{m}}{\partial y^{j}} & =-\frac{1}{2} \tilde{g}^{j k} \tilde{g}^{i p} \frac{\partial^{2} \tilde{g}_{i p}}{\partial y^{j} \partial y^{k}}+O\left(|y|^{-4}\right) \\
& =-\frac{1}{2} \Delta_{g} \log |\tilde{g}|+O\left(|y|^{-4}\right),
\end{aligned}
$$

which proves the lemma.

Corollary 5.4. If in addition $R=O\left(|x|^{-3-\tau}\right)$ for some $\tau>0$, then in the asymptotically harmonic coordinate $\left\{y^{i}\right\}$, we have

$$
\sum_{i=1}^{3} \tilde{h}_{i i}=8 m(g) /|y|+o\left(|y|^{-1-\tau / 2}\right) .
$$

Proof. First we know that

$$
\lim _{|x| \rightarrow \infty} \frac{|y|}{|x|}=1 .
$$


Then from Lemma 5.3, in the coordinate $\left\{y^{i}\right\}$ we have

$$
\Delta_{g} \log |\tilde{g}|=O\left(|y|^{-3-\tau}\right) .
$$

We know that

$$
\log |\tilde{g}|=O\left(|y|^{-1}\right) .
$$

From the theory of harmonic functions in $\mathbb{R}^{n}$, there exists a constant $C$ such that

$$
\log |\tilde{g}|=\frac{C}{|y|}+o\left(|y|^{-1-\tau / 2}\right) .
$$

From Bartnik's result, we know the mass is invariant under the change of coordinates because $R(g) \in L^{1}$ :

$$
m(g)=\lim _{R \rightarrow \infty} \frac{1}{16 \pi} \int_{s_{R}}\left(\tilde{h}_{i j, j}-\tilde{h}_{j j, i}\right) v_{g}^{i} d \mu .
$$

Now,

$$
\begin{aligned}
\tilde{g}_{i k, k}-\frac{1}{2} \tilde{g}_{k k, i} & =\tilde{g}^{i j} \tilde{g}^{k l}\left(\tilde{g}_{j k, l}-\frac{1}{2} \tilde{g}_{k l, j}\right)+O\left(|y|^{-3}\right) \\
& =-\Delta_{g} y^{i}+O\left(|y|^{-3}\right)=O\left(|y|^{-3}\right)
\end{aligned}
$$

therefore

$$
\begin{aligned}
m(g) & =\lim _{R \rightarrow \infty} \frac{1}{16 \pi} \int_{s_{R}}\left(-\frac{1}{2} \tilde{h}_{j j, i}\right) v_{g}^{i} d \mu \\
& =-\lim _{R \rightarrow \infty} \frac{1}{32 \pi} \int_{s_{R}} \frac{\partial \log |\tilde{g}|}{\partial y^{i}} v_{g}^{i} d \mu \\
& =\lim _{R \rightarrow \infty} \frac{1}{32 \pi} \int_{s_{R}} \frac{C y^{i}}{|y|^{3}} v_{g}^{i} d \mu \\
& =\frac{C}{8} .
\end{aligned}
$$

So we get the result by easy calculation.

Remark 5.5. We can replace the constraint equation by the condition

$$
R=O\left(|x|^{-3-\tau}\right) \quad \text { for some } \tau>0 .
$$

\section{Proof of Theorem 1.7}

Now let's prove Theorem 1.7. First note that, if it were false, we could find a sequence $\Sigma_{n}$ of stable constant mean curvature surfaces with $r_{0}\left(\Sigma_{n}\right) \rightarrow \infty$ and $\log r_{1}\left(\Sigma_{n}\right) \leq \frac{1}{n} r_{0}\left(\Sigma_{n}\right)^{1 / 4}$; but the $\Sigma_{n}$ do not belong to the foliation constructed by the standard method. So $r_{1} \leq C r_{0}$ cannot hold with a uniform $C$, by Huang's uniqueness theorem. 
Recall that, for any surface $\Sigma$ embedded in $\mathbb{R}^{3}$ and any given vector $b \in \mathbb{R}^{3}$,

$$
\int_{\Sigma} H_{e}\left\langle v_{e} \cdot b\right\rangle_{e} d \mu_{e}=0
$$

where $H_{e}$ and $v_{e}$ denote the mean curvature and normal vector field with respect to the Euclidean metric.

On the other hand, if $\Sigma$ is a constant mean curvature surface in the asymptotically flat end, then

So we have

$$
\int_{\Sigma} H\left\langle v_{e} \cdot b\right\rangle_{e} d \mu_{e}=0
$$

$$
\int_{\Sigma}\left(H-H_{e}\right)\left\langle v_{e} \cdot b\right\rangle_{e} d \mu_{e}=0 .
$$

Now we want to prove that, for a sequence of stable constant mean curvature spheres $\Sigma_{n}$ with $r_{0}\left(\Sigma_{n}\right) \rightarrow \infty$ and $\log r_{1}\left(\Sigma_{n}\right) \leq \frac{1}{n} r_{0}\left(\Sigma_{n}\right)^{1 / 4}$, if there does not exist a uniform constant $C$ such that $r_{1} \leq C r_{0}$ for every $\Sigma_{n}$, then there exists a subsequence (also denoted by $\Sigma_{n}$ ) and a constant vector $b$, such that

$$
\limsup _{n \rightarrow \infty} \int_{\Sigma_{n}}\left(H-H_{e}\right)\left\langle v_{e}, b\right\rangle_{e} d \mu_{e}<0 .
$$

Then Theorem 1.7 follows from this contradiction.

From now on, our calculation is in the asymptotically harmonic coordinate $\left\{x^{i}\right\}$. We have calculated $H-H_{e}$, so

$$
\begin{aligned}
\int_{\Sigma}(H- & \left.H_{e}\right)\left\langle v_{e} \cdot b\right\rangle_{e} d \mu_{e} \\
= & \int_{\Sigma}\left(-f^{i k} h_{k l} f^{l j} A_{i j}+\frac{1}{2} H v^{i} v^{j} h_{i j}-f^{i j} v^{l} \bar{\nabla}_{i} h_{j l}\right. \\
& \left.+\frac{1}{2} f^{i j} v^{l} \bar{\nabla}_{l} h_{i j} \pm C|h||\bar{\nabla} h| \pm C|h|^{2}|A|\right)\left\langle v_{e} \cdot b\right\rangle_{e} d \mu_{e} .
\end{aligned}
$$

For the sequence of constant mean curvature surfaces $\Sigma_{n}$ chosen above, we have

$$
\lim _{n \rightarrow \infty} r_{0}\left(\Sigma_{n}\right)=\infty, \quad \lim _{n \rightarrow \infty} H\left(\Sigma_{n}\right) r_{0}\left(\Sigma_{n}\right)=0
$$

and

$$
\lim _{n \rightarrow \infty} \frac{\log r_{1}\left(\Sigma_{n}\right)}{r_{0}\left(\Sigma_{n}\right)^{1 / 4}}=0
$$

because all the radius conditions are preserved when the coordinates turn into asymptotically harmonic coordinates.

So we can choose $s$ sufficiently small and $K$ sufficiently large with $s H^{-1}>K r_{0}$ for $r_{0}$ sufficiently large. 
We know that

$$
|h|=O\left(|x|^{-1}\right), \quad|\bar{\nabla} h|=O\left(|x|^{-2}\right), \quad|A| \leq C H+C|\AA| .
$$

From the estimate

$$
|\AA| \leq r_{0}^{-1 / 2} O\left(|x|^{-1}\right),
$$

we have

$$
\left|\int_{\Sigma}\left( \pm C|h||\bar{\nabla} h| \pm C|h|^{2}|A|\right)\left\langle v_{e} \cdot b\right\rangle_{e} d \mu_{e}\right| \leq C \int_{\Sigma}\left(H|x|^{-2}+|x|^{-3}\right)=O\left(r_{0}^{-1}\right)
$$

by the estimates in Section 2.

Now we calculate other terms in (6-2):

$$
\begin{aligned}
\int_{\Sigma_{n}}-f^{i j} v^{l}\left(\bar{\nabla}_{i} h_{j l}\right) v^{m} b^{m} d \mu_{e} & \\
=\frac{1}{2} \int_{\Sigma_{n}}\left(f^{i j} h_{j k} f^{k l} A_{l i}-H v^{j} v^{l} h_{j l}\right) v^{m} b^{m} d \mu_{e} & +\frac{1}{2} \int_{\Sigma_{n}} f^{i j} v^{l} h_{j l} A_{i k} f^{k m} b^{m} d \mu_{e} \\
& -\frac{1}{2} \int_{\Sigma_{n}} f^{i j} v^{l}\left(\bar{\nabla}_{i} h_{j l}\right) v^{m} b^{m} d \mu_{e}
\end{aligned}
$$

because $d \mu_{e}=\left(1+O\left(r^{-1}\right)\right) d \mu, v_{e}=\left(1+O\left(r^{-1}\right)\right) v$ and $\left\langle v_{e} \cdot b\right\rangle_{e}=\langle v \cdot b\rangle_{g}+O\left(r^{-1}\right)$.

So we have

$$
\begin{aligned}
& \int_{\Sigma_{n}}\left(H-H_{e}\right)\left\langle v_{e} \cdot b\right\rangle_{e} d \mu_{e} \\
&=\int_{\Sigma_{n}}-\frac{1}{2} f^{i k} h_{k l} f^{l j} A_{i j} v^{m} b^{m}+f^{i j} v^{l} h_{j l} A_{i k} f^{k m} b^{m} \\
& \quad-\frac{1}{2} f^{i j} v^{l} \bar{\nabla}_{i} h_{j l} v^{m} b^{m}+\frac{1}{2} f^{i j} v^{l} \bar{\nabla}_{l} h_{i j} v^{m} b^{m}+O\left(r_{0}^{-1}\right) d \bar{\mu} .
\end{aligned}
$$

Note that

$$
A_{i j}=\AA_{i j}+\frac{f_{i j}}{2} H, \quad \sup |\AA| \leq r_{0}^{-1 / 2} O\left(|x|^{-1}\right),
$$

hence

$$
\begin{aligned}
& \int_{\Sigma_{n}}\left(H-H_{e}\right)\left\langle v_{e} \cdot b\right\rangle_{e} d \mu_{e}=\int_{\Sigma_{n}}-\frac{H}{4} f^{k l} h_{k l} v^{m} b^{m}+\frac{H}{4} f^{j m} h_{j l} v^{l} b^{m} \\
& +\frac{1}{2} f^{i j}\left(\bar{\nabla}_{l} h_{i j}\right) v^{l} v^{m} b^{m}-\frac{1}{2} f^{i j}\left(\bar{\nabla}_{i} h_{j l}\right) v^{l} v^{m} b^{m} \\
& \pm C \int_{\Sigma_{n}}|x|^{-2} r_{0}^{-1 / 2}+O\left(r_{0}^{-1}\right) .
\end{aligned}
$$

In this case we calculate

$$
\int_{\Sigma_{n}}|x|^{-2} r_{0}^{-1 / 2} d \mu_{e}
$$


We divide the integral into three parts:

$$
\int_{\Sigma_{n}}|x|^{-2} r_{0}^{-1 / 2}=\int_{\Sigma_{n} \cap B_{s H^{-1}}^{c}(0)}+\int_{\Sigma_{n} \cap B_{K r_{0}}(0)}+\int_{\Sigma_{n} \cap\left(B_{\left.s H^{-1} \backslash B_{K r_{0}}\right)}\right.}|x|^{-2} r_{0}^{-1 / 2} .
$$

Then by the blowdown results in Section 3,

$$
\begin{aligned}
& \int_{\Sigma_{n} \cap B_{s H^{-1}}^{c}(0)}|x|^{-2} r_{0}^{-1 / 2} d \mu_{e}=\int_{\tilde{\Sigma}_{n} \cap B_{s}^{c}(0)}|\tilde{x}|^{-2} r_{0}^{-1 / 2} d \tilde{\mu} \leq C r_{0}^{-1 / 2} \\
& \int_{\Sigma_{n} \cap B_{K r_{0}}(0)}|x|^{-2} r_{0}^{-1 / 2} d \mu_{e}=\int_{\hat{\Sigma}_{n} \cap B_{k}(0)}|\hat{x}|^{-2} r_{0}^{-1 / 2} d \hat{\mu} \leq C r_{0}^{-1 / 2}
\end{aligned}
$$

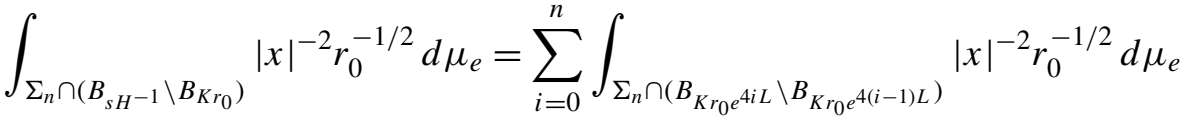

$$
\begin{aligned}
& \leq C \sum_{i=0}^{n} \int_{B_{e^{4 L} \backslash B_{1}}}|\bar{x}|^{-2} r_{0}^{-1 / 2} d \bar{\mu} \leq C r_{0}^{-1 / 2} l_{n} L,
\end{aligned}
$$

where $e^{l_{n} L} K r_{0}=s H^{-1}$.

So if

$$
\lim _{r_{0} \rightarrow 0} \frac{|\log H|}{r_{0}^{1 / 2}}=0
$$

in other words,

we have

$$
\lim _{r_{0} \rightarrow 0} \frac{\left|\log r_{1}\right|}{r_{0}^{1 / 2}}=0,
$$

$$
\int_{\Sigma}|x|^{-2} r_{0}^{-1 / 2} d \bar{\mu} \rightarrow 0
$$

as $r_{0} \rightarrow \infty$.

From the property of the asymptotically harmonic coordinate,

$$
\begin{gathered}
g^{i j} h_{i j}=\frac{8 m(g)}{r}+o\left(r^{-1-\tau / 2}\right), \\
g^{k l}\left(g_{i k, l}-\frac{1}{2} g_{k l, i}\right)=O\left(|x|^{-3}\right), \\
\int_{\Sigma_{n}}-\frac{H}{4} f^{k l} h_{k l} v^{m} b^{m}+\frac{H}{4} f^{j m} h_{j l} v^{l} b^{m}+\frac{1}{2} f^{i j}\left(\bar{\nabla}_{l} h_{i j}-\bar{\nabla}_{i} h_{j l}\right) v^{l} v^{m} b^{m} \\
=\int_{\Sigma_{n}}-\frac{H}{4} g^{k l} h_{k l} v^{m} b^{m}+\frac{H}{4} g^{j m} h_{j l} v^{l} b^{m} \\
+\frac{1}{2} g^{i j}\left(\bar{\nabla}_{l} h_{i j}-\bar{\nabla}_{i} h_{j l}\right) v^{l} v^{m} b^{m}+O\left(\left|r_{0}\right|^{-1}\right) \\
=-2 m(g) \int_{\Sigma_{n}}\left(\frac{H}{r}\left\langle v_{e} \cdot b_{e}\right\rangle_{e}+\frac{\left\langle x \cdot v_{e}\right\rangle_{e}\left\langle v_{e} \cdot b\right\rangle_{e}}{r^{3}}\right)+\int_{\Sigma_{n}} \frac{H}{4} h_{m l} v^{l} b^{m}+o(1) .
\end{gathered}
$$


Therefore,

$$
\lim _{n \rightarrow \infty}\left(-2 m(g) \int_{\Sigma_{n}}\left(\frac{H}{r}\left\langle v_{e} \cdot b\right\rangle_{e}+\frac{\left\langle x \cdot v_{e}\right\rangle_{e}\left\langle v_{e} \cdot b\right\rangle_{e}}{r^{3}}\right)+\int_{\Sigma_{n}} \frac{H}{4} h_{m l} v^{l} b^{m}\right)=0 .
$$

Note that

$$
h_{m l} v^{l}=\left(h_{m l}-\frac{\operatorname{tr}(h)}{2} \delta_{m l}\right) v^{l}+\frac{\operatorname{tr}(h)}{2} v^{m},
$$

where $\operatorname{tr}(h)=g^{i j} h_{i j}$.

Assume that the three eigenvalues of $h_{m l}$ are

$$
\lambda_{1} \geq \lambda_{2} \geq \lambda_{3} \geq 0
$$

For $p \in \Sigma$ fixed, choose coordinates properly so

$$
h_{m l}-\frac{\operatorname{tr}(h)}{2} \delta_{m l}
$$

can be written as

$$
\left(\begin{array}{ccc}
\lambda_{1}-\frac{\operatorname{tr}(h)}{2} & 0 & 0 \\
0 & \lambda_{2}-\frac{\operatorname{tr}(h)}{2} & 0 \\
0 & 0 & \lambda_{3}-\frac{\operatorname{tr}(h)}{2}
\end{array}\right) .
$$

Assume $v=\left(\tilde{v}^{1}, \tilde{v}^{2}, \tilde{v}^{3}\right)$ and $\left(\tilde{v}^{1}\right)^{2}+\left(\tilde{v}^{2}\right)^{2}+\left(\tilde{v}^{3}\right)^{2}=1$. Then

$$
\sum_{i=1}^{3}\left(\left(\lambda_{i}-\frac{\operatorname{tr}(h)}{2}\right) \tilde{v}^{i}\right)^{2}=\frac{(\operatorname{tr}(h))^{2}}{4}-\sum_{i=1}^{3} \lambda_{i}\left(\operatorname{tr}(h)-\lambda_{i}\right)\left(\tilde{v}^{i}\right)^{2} .
$$

Because of uniform ellipticity, there exists $C>0$ such that

so

$$
\frac{\operatorname{tr}(h)}{C} \leq \lambda_{3} \leq \lambda_{2} \leq \lambda_{1} \leq\left(1-\frac{1}{C}\right) \operatorname{tr}(h),
$$

Hence

$$
\lambda_{i}\left(\operatorname{tr}(h)-\lambda_{i}\right) \geq \frac{1}{C}\left(1-\frac{1}{C}\right)(\operatorname{tr}(h))^{2} .
$$

$$
\begin{aligned}
\sum_{i=1}^{3}\left(\left(\lambda_{i}-\frac{\operatorname{tr}(h)}{2}\right) \tilde{v}^{i}\right)^{2} & \leq\left(\frac{1}{4}-\frac{1}{C}\left(1-\frac{1}{C}\right)\right)(\operatorname{tr}(h))^{2} \\
\int_{\Sigma_{n}} \frac{H}{4} h_{m l} v^{l} b^{m} & =\int_{\Sigma_{n}} \frac{H}{4}\left(\frac{\operatorname{tr}(h)}{2}(v \cdot b)+\left(h_{m l}-\frac{\operatorname{tr}(h)}{2} \delta_{m l}\right) v^{l} b^{m}\right) \\
& \leq \int_{\Sigma_{n}} \frac{H \operatorname{tr}(h)}{4}\left(\frac{1}{2}(v \cdot b)+\sqrt{\frac{1}{4}-\frac{1}{C}\left(1-\frac{1}{C}\right)}\right) \\
& =\int_{\Sigma_{n}} \frac{H m(g)}{r}\left((v \cdot b)+1-\frac{2}{C}\right) .
\end{aligned}
$$


Thus, as $n \rightarrow \infty$,

$$
\begin{aligned}
& \int_{\Sigma_{n}}\left(H-H_{e}\right)\left\langle v_{e} \cdot b\right\rangle_{e} \\
& \quad \leq-m \int_{\Sigma_{n}} \frac{H}{r}\left\langle v_{e} \cdot b\right\rangle_{e}+\frac{2}{r^{3}}\left\langle x \cdot v_{e}\right\rangle_{e}\left\langle v_{e} \cdot b\right\rangle_{e} d \mu_{e}+\left(1-\frac{2}{C}\right) m(g) \int_{\Sigma_{n}} \frac{H}{r} d \mu_{e}+o(1) .
\end{aligned}
$$

From Lemma 3.1, we have that $(H / 2) \Sigma_{n}$ subconverges to some sphere $S_{1}^{2}(a)$ with $|a|=1$. Now we choose $b=-a$. Then by the calculation in [Qing and Tian 2007],

$$
\begin{aligned}
- & m(g) \int_{\Sigma_{n}} \frac{H}{r}\left\langle v_{e} \cdot b\right\rangle_{e} \\
-m(g) \int_{\Sigma_{n}} \frac{2}{r^{3}}\left\langle x \cdot v_{e}\right\rangle_{e}\left\langle v_{e} \cdot b\right\rangle_{e} & \rightarrow-\frac{8}{3} \pi m(g) \\
\left(1-\frac{2}{C}\right) m(g) \int_{\Sigma_{n}} \frac{H}{r} & \rightarrow\left(1-\frac{2}{C}\right) 8 \pi m(g)
\end{aligned}
$$

as $n \rightarrow \infty$.

Since there is a small difference from [Qing and Tian 2007], we prove these convergences again. Notice from Lemma 3.1 that $(H / 2) \Sigma_{n}$ subconverges to some sphere $S_{1}(a)$ with $|a|=1$, and the first and third integral converge, respectively, to

$-m(g) \int_{S_{1}(a)} \frac{2}{r}\left\langle v_{e} \cdot b\right\rangle_{e}=-\frac{8}{3} \pi m(g)$ and $\left(1-\frac{2}{C}\right) m(g) \int_{S_{1}(a)} \frac{2}{r}=\left(1-\frac{2}{C}\right) 8 \pi m(g)$.

To deal with (6-4), first notice that

$$
\int_{S^{2}(a)} \frac{2}{r^{3}}\left\langle x \cdot v_{e}\right\rangle_{e}\left\langle v_{e} \cdot b\right\rangle_{e} d \mu_{e}=\frac{4}{3} \pi .
$$

Then we break up the integral (6-4) into three parts:

$$
\begin{aligned}
\int_{\Sigma_{n}} \frac{2}{r^{3}}\left\langle x \cdot v_{e}\right\rangle_{e}\left\langle v_{e} \cdot b\right\rangle_{e} d \mu_{e} & \\
= & \int_{\Sigma_{n} \cap B_{s H^{-1}}^{c}(0)}+\int_{\Sigma_{n} \cap B_{K r_{0}}(0)}+\int_{\Sigma_{n} \cap B_{s H^{-1} \backslash B_{K r_{0}}}} \frac{2}{r^{3}}\left\langle x \cdot v_{e}\right\rangle_{e}\left\langle v_{e} \cdot b\right\rangle_{e} d \mu_{e} .
\end{aligned}
$$

Then

$$
\begin{aligned}
& \lim _{n \rightarrow \infty} \int_{\Sigma_{n} \cap B_{s H^{c}}^{c}(0)} \frac{2}{r^{3}}\left\langle x \cdot v_{e}\right\rangle_{e}\left\langle v_{e} \cdot b\right\rangle_{e} d \mu_{e}=\int_{S^{2}(a) \cap B_{s}^{c}} \frac{2}{r^{3}}\left\langle x \cdot v_{e}\right\rangle_{e}\left\langle v_{e} \cdot b\right\rangle_{e} d \mu_{e}, \\
& \lim _{n \rightarrow \infty} \int_{\Sigma_{n} \cap B_{K r_{0}}(0)} \frac{2}{r^{3}}\left\langle x \cdot v_{e}\right\rangle_{e}\left\langle v_{e} \cdot b\right\rangle_{e} d \mu_{e}=\int_{P \cap B_{K}(0)} \frac{2}{r^{3}}\left\langle x \cdot v_{e}\right\rangle_{e}\left\langle v_{e} \cdot b\right\rangle_{e} d \mu_{e}
\end{aligned}
$$


where $P$ is the limit plane in Lemma 3.2. From Corollary 4.7, the normal vector of $P$ is $v_{e}$. Due to an easy calculation,

$$
\int_{P} \frac{2}{r^{3}}\left\langle x \cdot v_{e}\right\rangle_{e}\left\langle v_{e} \cdot b\right\rangle_{e} d \mu_{e}=4 \pi
$$

From the divergence theorem,

$$
\int_{\Sigma_{n}} \frac{2}{r^{3}}\left\langle x \cdot v_{e}\right\rangle_{e} d \mu_{e}=8 \pi
$$

for any $n$ and

$$
\int_{S^{2}(a)} \frac{2}{r^{3}}\left\langle x \cdot v_{e}\right\rangle_{e} d \mu_{e}=4 \pi,
$$

because the origin is on the sphere $S^{2}(a)$. Since

$$
\begin{aligned}
& \lim _{n \rightarrow \infty} \int_{\Sigma_{n} \cap B_{s H^{-1}}^{c}(0)} \frac{2}{r^{3}}\left\langle x \cdot v_{e}\right\rangle_{e} d \mu_{e}=\int_{S^{2}(a) \cap B_{s}^{c}(0)} \frac{2}{r^{3}}\left\langle x \cdot v_{e}\right\rangle_{e} d \mu_{e}, \\
& \lim _{n \rightarrow \infty} \int_{\Sigma_{n} \cap B_{K r_{0}}(0)} \frac{\frac{2}{r^{3}}\left\langle x \cdot v_{e}\right\rangle_{e} d \mu_{e}}{r^{3}}=\int_{P \cap B_{K}(0)} \frac{2}{r^{3}}\left\langle x \cdot v_{e}\right\rangle_{e} d \mu_{e}, \\
& \int_{P} \frac{2}{r^{3}}\left\langle x \cdot v_{e}\right\rangle_{e} d \mu_{e}=4 \pi,
\end{aligned}
$$

we have

$$
\lim _{s \rightarrow 0, K \rightarrow \infty} \limsup _{n \rightarrow \infty}\left|\int_{\Sigma_{n} \cap\left(B_{s H^{-1}} \backslash B_{K r_{0}}\right)} \frac{2}{r^{3}}\left\langle x \cdot v_{e}\right\rangle_{e} d \mu_{e}\right|=0 .
$$

Now we want to prove that

$$
\lim _{s \rightarrow 0, K \rightarrow \infty} \limsup _{n \rightarrow \infty}\left|\int_{\Sigma_{n} \cap\left(B_{\left.s H^{-1} \backslash B_{K r_{0}}\right)}\right.} \frac{2}{r^{3}}\left\langle x \cdot v_{e}\right\rangle_{e}\left\langle v_{e} \cdot b\right\rangle_{e} d \mu_{e}\right|=0 .
$$

Use Corollary 4.8 to get (6-5) from (6-4), but there is a small difference from [Qing and Tian 2007]:

$$
\begin{aligned}
\int_{\Sigma_{n} \cap\left(B_{\left.s H^{-1} \backslash B_{K r_{0}}\right)} \frac{2}{r^{3}}\left\langle x \cdot v_{e}\right\rangle_{e}\left\langle v_{e} \cdot b\right\rangle_{e} d \mu_{e}\right.} & \\
=\left\langle v_{n} \cdot b\right\rangle_{e} & \int_{\Sigma_{n} \cap\left(B_{\left.s H^{-1} \backslash B_{K r_{0}}\right)}\right.} \frac{2}{r^{3}}\left\langle v_{e} \cdot b\right\rangle_{e} d \mu_{e} \\
& +\int_{\Sigma_{n} \cap\left(B_{\left.s H^{-1} \backslash B_{K r_{0}}\right)}\right.} \frac{2}{r^{3}}\left\langle x \cdot v_{e}\right\rangle_{e}\left\langle\left(v_{e}-v_{n}\right) \cdot b\right\rangle_{e} d \mu_{e} .
\end{aligned}
$$


The first term will converge to 0 . We deal with the second term using the cylindrical coordinates in Section 4:

$$
\begin{aligned}
\left|\int_{\Sigma_{n} \cap\left(B_{s H^{-1}} \backslash B_{K r_{0}}\right)} \frac{2}{r^{3}}\left\langle x \cdot v_{e}\right\rangle_{e}\left\langle\left(v_{e}-v_{n}\right) \cdot b\right\rangle_{e} d \mu_{e}\right| \\
=\left|\sum_{j=1}^{l_{n}} \int_{A_{K r_{0} e^{(j-1) L}, K r_{0} e^{j L}}} \frac{2}{r^{3}}\left\langle x \cdot v_{e}\right\rangle_{e}\left\langle\left(v_{e}-v_{n}\right) \cdot b\right\rangle_{e} d \mu_{e}\right| \\
\leq C \sum_{j=1}^{l_{n}} L \max _{I_{j}}\left|v_{e}-v_{n}\right| \\
=C \sum_{j=1}^{l_{n} / 2} L \max _{I_{j}}\left|v_{e}-v_{n}\right|+C \sum_{j=l_{n} / 2+1}^{l_{n}} L \max _{I_{j}}\left|v_{e}-v_{n}\right| .
\end{aligned}
$$

From Corollary 4.8,

$$
C L \sum_{i=1}^{l_{n} / 2} \sup _{I_{i}}\left|v-v_{n}\right|+C L \sum_{i=l_{n} / 2+1}^{l_{n}} \sup _{I_{i}}\left|v-v_{n}\right| \leq C\left(l_{n} e^{-(1 / 4) l_{n} L}+C\right) s+l_{n}^{2} r_{0}^{-1 / 2} .
$$

But from the condition

$$
\lim _{n \rightarrow \infty} \frac{\log r_{1}\left(\Sigma_{n}\right)}{r_{0}\left(\Sigma_{n}\right)^{1 / 4}}=0
$$

we know

$$
\lim _{n \rightarrow \infty} l_{n}^{2} r_{0}^{-1 / 2}=\lim _{n \rightarrow \infty}\left(\frac{L^{-1}\left(\log s H^{-1}-\log K r_{0}\right)}{r_{0}^{1 / 4}}\right)^{2}=0,
$$

so (6-5) holds.

Then

$$
0 \leq-\frac{8}{3} \pi m(g)-\frac{16}{3} \pi m(g)+\left(1-\frac{2}{C}\right) 8 \pi m(g)=-\frac{16}{C} \pi m(g) .
$$

But $m(g)>0$, so

$$
\limsup _{n \rightarrow \infty} \int_{\Sigma_{n}}\left(H-H_{e}\right)\left\langle v_{e}, b\right\rangle_{e} d \mu_{e}<0 .
$$

This proves Theorem 1.7.

\section{References}

[Bartnik 1986] R. Bartnik, "The mass of an asymptotically flat manifold", Comm. Pure Appl. Math. 39:5 (1986), 661-693. MR 88b:58144 Zbl 0598.53045

[Huang 2009] L.-H. Huang, "On the center of mass of isolated systems with general asymptotics", Classical Quantum Gravity 26:1 (2009), 015012, 25. MR 2010e:83009 Zbl 1157.83011 
[Huang 2010] L.-H. Huang, "Foliations by stable spheres with constant mean curvature for isolated systems with general asymptotics", Comm. Math. Phys. 300:2 (2010), 331-373. MR 2728728 Zbl 1206.53028

[Huisken and Ilmanen 2001] G. Huisken and T. Ilmanen, "The inverse mean curvature flow and the Riemannian Penrose inequality", J. Differential Geom. 59:3 (2001), 353-437. MR 2003h:53091 Zbl 1055.53052

[Huisken and Yau 1996] G. Huisken and S.-T. Yau, "Definition of center of mass for isolated physical systems and unique foliations by stable spheres with constant mean curvature", Invent. Math. 124:1-3 (1996), 281-311. MR 96m:53037 Zbl 0858.53071

[Kenmotsu 2003] K. Kenmotsu, Surfaces with constant mean curvature, Transl. of Math. Monogr. 221, Amer. Math. Soc., Providence, RI, 2003. MR 2004m:53014 Zbl 1042.53001

[Li and Yau 1982] P. Li and S. T. Yau, "A new conformal invariant and its applications to the Willmore conjecture and the first eigenvalue of compact surfaces", Invent. Math. 69:2 (1982), 269-291. MR 84f:53049 Zbl 0503.53042

[Michael and Simon 1973] J. H. Michael and L. M. Simon, "Sobolev and mean-value inequalities on generalized submanifolds of $R^{n}$ ", Comm. Pure Appl. Math. 26 (1973), 361-379. MR 0344978 (49 \#9717

[Qing and Tian 1997] J. Qing and G. Tian, "Bubbling of the heat flows for harmonic maps from surfaces”, Comm. Pure Appl. Math. 50:4 (1997), 295-310. MR 98k:58070 Zbl 0879.58017

[Qing and Tian 2007] J. Qing and G. Tian, "On the uniqueness of the foliation of spheres of constant mean curvature in asymptotically flat 3-manifolds", J. Amer. Math. Soc. 20:4 (2007), 1091-1110. MR 2008g:53034 Zbl 1142.53024

[Schoen et al. 1975] R. Schoen, L. Simon, and S. T. Yau, "Curvature estimates for minimal hypersurfaces”, Acta Math. 134:3-4 (1975), 275-288. MR 54 \#11243 Zbl 0323.53039

[Simon 1993] L. Simon, "Existence of surfaces minimizing the Willmore functional", Comm. Anal. Geom. 1:2 (1993), 281-326. MR 94k:58028 Zbl 0848.58012

Received August 19, 2010.

SHIGUANG MA

SCHOOL OF MATHEMATICAL SCIENCES

PEKING UNIVERSITY

BEIJING, 100871

CHINA

msgdyx8741@163.com 


\title{
PACIFIC JOURNAL OF MATHEMATICS
}

\author{
http://www.pjmath.org \\ Founded in 1951 by
}

E. F. Beckenbach (1906-1982) and F. Wolf (1904-1989)

\section{EDITORS}

V. S. Varadarajan (Managing Editor)

Department of Mathematics

University of California

Los Angeles, CA 90095-1555

pacific@math.ucla.edu

Vyjayanthi Chari

Department of Mathematics

University of California

Riverside, CA 92521-0135

chari@math.ucr.edu

\section{Robert Finn}

Department of Mathematics Stanford University

Stanford, CA 94305-2125

finn@math.stanford.edu

Kefeng Liu

Department of Mathematics

University of California

Los Angeles, CA 90095-1555

liu@math.ucla.edu
Darren Long

Department of Mathematics

University of California

Santa Barbara, CA 93106-3080

long@math.ucsb.edu

Jiang-Hua Lu

Department of Mathematics

The University of Hong Kong

Pokfulam Rd., Hong Kong jhlu@maths.hku.hk

Alexander Merkurjev

Department of Mathematics University of California

Los Angeles, CA 90095-1555 merkurev@math.ucla.edu
Sorin Popa

Department of Mathematics

University of California

Los Angeles, CA 90095-1555

popa@math.ucla.edu

Jie Qing

Department of Mathematics

University of California

Santa Cruz, CA 95064

qing@ cats.ucsc.edu

Jonathan Rogawski

Department of Mathematics

University of California

Los Angeles, CA 90095-1555

jonr@math.ucla.edu

\section{PRODUCTION}

pacific@math.berkeley.edu

Silvio Levy, Scientific Editor Matthew Cargo, Senior Production Editor

ACADEMIA SINICA, TAIPEI

CALIFORNIA INST. OF TECHNOLOGY

INST. DE MATEMÁTICA PURA E APLICADA

KEIO UNIVERSITY

MATH. SCIENCES RESEARCH INSTITUTE

NEW MEXICO STATE UNIV.

OREGON STATE UNIV.

\section{SUPPORTING INSTITUTIONS}

STANFORD UNIVERSITY
UNIV. OF BRITISH COLUMBIA
UNIV. OF CALIFORNIA, BERKELEY
UNIV. OF CALIFORNIA, DAVIS
UNIV. OF CALIFORNIA, LOS ANGELES
UNIV. OF CALIFORNIA, RIVERSIDE
UNIV. OF CALIFORNIA, SAN DIEGO
UNIV. OF CALIF., SANTA BARBARA

UNIV. OF CALIF., SANTA CRUZ

UNIV. OF MONTANA

UNIV. OF OREGON

UNIV. OF SOUTHERN CALIFORNIA

UNIV. OF UTAH

UNIV. OF WASHINGTON

WASHINGTON STATE UNIVERSITY

These supporting institutions contribute to the cost of publication of this Journal, but they are not owners or publishers and have no responsibility for its contents or policies.

See inside back cover or www.pjmath.org for submission instructions.

The subscription price for 2011 is US \$420/year for the electronic version, and \$485/year for print and electronic.

Subscriptions, requests for back issues from the last three years and changes of subscribers address should be sent to Pacific Journal of Mathematics, P.O. Box 4163, Berkeley, CA 94704-0163, U.S.A. Prior back issues are obtainable from Periodicals Service Company, 11 Main Street, Germantown, NY 12526-5635. The Pacific Journal of Mathematics is indexed by Mathematical Reviews, Zentralblatt MATH, PASCAL CNRS Index, Referativnyi Zhurnal, Current Mathematical Publications and the Science Citation Index.

The Pacific Journal of Mathematics (ISSN 0030-8730) at the University of California, c/o Department of Mathematics, 969 Evans Hall, Berkeley, CA 94720-3840, is published monthly except July and August. Periodical rate postage paid at Berkeley, CA 94704, and additional mailing offices. POSTMASTER: send address changes to Pacific Journal of Mathematics, P.O. Box 4163, Berkeley, CA 94704-0163.

PJM peer review and production are managed by EditFLOW ${ }^{\mathrm{TM}}$ from Mathematical Sciences Publishers.

PUBLISHED BY PACIFIC JOURNAL OF MATHEMATICS

at the University of California, Berkeley 94720-3840

A NON-PROFIT CORPORATION

Typeset in IATEX

Copyright $(2011$ by Pacific Journal of Mathematics 


\section{PACIFIC JOURNAL OF MATHEMATICS}

Volume $252 \quad$ No. $1 \quad$ July 2011

Some dynamic Wirtinger-type inequalities and their applications

RaVi P. Agarwal, Martin Bohner, Donal O'REgAn and SAMIR H.

SAKER

Splitting criteria for vector bundles on higher-dimensional varieties

PARSA BAKHTARY

Average Mahler's measure and $L_{p}$ norms of unimodular polynomials

KWOK-KWOng STEPHen CHOI and Michael J. MossinghofF

Tate resolutions and Weyman complexes

David A. CoX and EVgeny Materov

On pointed Hopf algebras over dihedral groups

FERNANDO FANTINO and GASTON ANDRÉS GARCIA

Integral topological quantum field theory for a one-holed torus

PATRicK M. Gilmer and Gregor Masbaum

Knot 4-genus and the rank of classes in $\boldsymbol{W}(\mathbb{Q}(t))$

CHARLES LIVINGSTON

Roots of Toeplitz operators on the Bergman space

ISSAM LOUHICHI and NAGISETTY V. RAO

Uniqueness of the foliation of constant mean curvature spheres in asymptotically 145 flat 3-manifolds

SHIGUANG MA

On the multiplicity of non-iterated periodic billiard trajectories

MARCo MAZZUCCHELLI

A remark on Einstein warped products

MiCHELE RIMOLDI

Exceptional Dehn surgery on large arborescent knots

YING-QING WU

Harnack estimates for the linear heat equation under the Ricci flow 